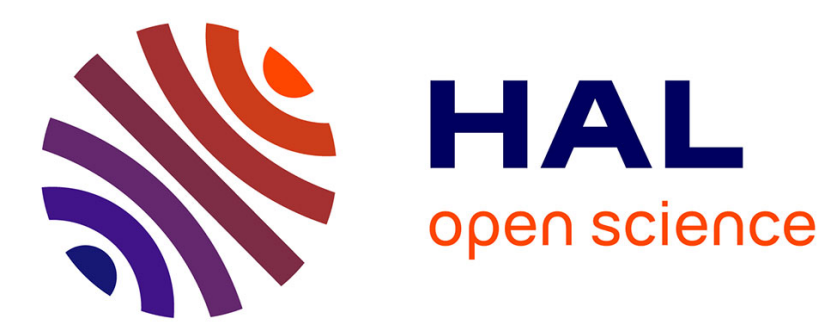

\title{
Micro- and Nanoscopic Imaging of Enzymatic Electrodes: A Review
}

Dodzi Zigah, Elisabeth Lojou, Anne Poulpiquet

\section{To cite this version:}

Dodzi Zigah, Elisabeth Lojou, Anne Poulpiquet. Micro- and Nanoscopic Imaging of Enzymatic Electrodes: A Review. ChemElectroChem, 2019, 6 (22), pp.5524-5546. 10.1002/celc.201901065 . hal02375017

\section{HAL Id: hal-02375017 \\ https://hal.science/hal-02375017}

Submitted on 21 Nov 2019

HAL is a multi-disciplinary open access archive for the deposit and dissemination of scientific research documents, whether they are published or not. The documents may come from teaching and research institutions in France or abroad, or from public or private research centers.
L'archive ouverte pluridisciplinaire HAL, est destinée au dépôt et à la diffusion de documents scientifiques de niveau recherche, publiés ou non, émanant des établissements d'enseignement et de recherche français ou étrangers, des laboratoires publics ou privés. 
Micro- and nanoscopic imaging of enzymatic electrodes: a review

Dr. Dodzi Zigah, ${ }^{1}$ Dr. Elisabeth Lojou, ${ }^{2}$ Dr. Anne de Poulpiquet*2

${ }^{1}$ Univ. Bordeaux, CNRS, Bordeaux INP, ISM, UMR 5255, 33400 Talence, France

${ }^{2}$ Aix-Marseille Univ., CNRS, BIP, UMR 7281, 31 Chemin Aiguier, 13009 Marseille, France

* Corresponding author: Dr. Anne de Poulpiquet. Tel: +33(0)491164144. Mail: adepoulpiquet@imm.cnrs.fr 


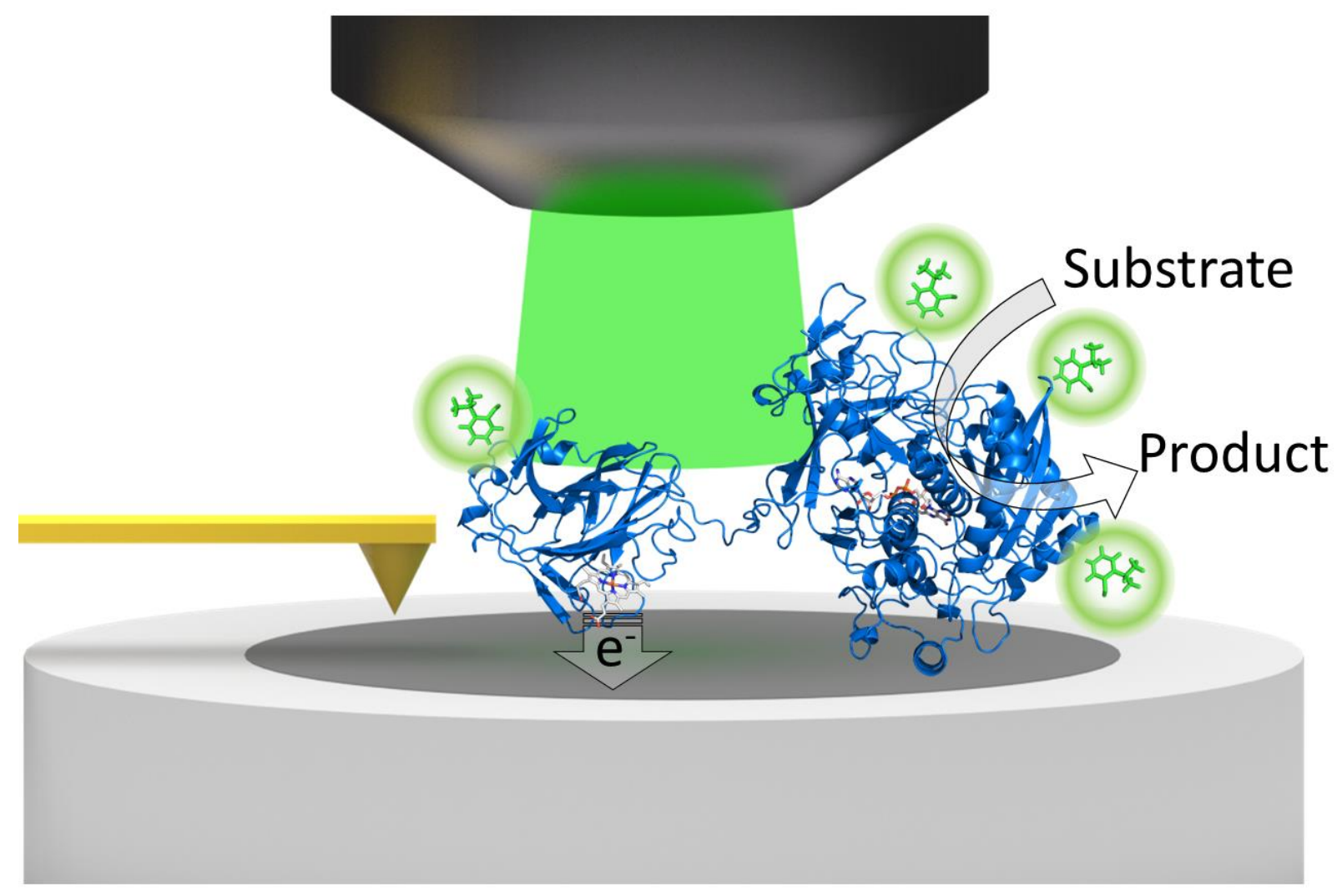




\section{Author biographies}

Dr. Dodzi Zigah has a Master degree in electrochemistry from the University Denis Diderot (Paris, France). In 2009, he obtained a Ph.D. in electrochemistry from the University of Rennes (France). After a postdoc position at the University of Texas in Austin, he joined the faculty of the University of Bordeaux (France) in 2010 as an assistant professor. His current research focuses on electrochemistry and surface modification. $\mathrm{He}$ is a specialist in electrochemical microscopy and bipolar electrochemistry, to prepare and analyze hybrid materials in the fields of photoelectrochemistry and electroanalysis.

Dr. Elisabeth Lojou is research director at the CNRS, France. She obtained her degree in engineering from the National School of Chemistry, Rennes, France in 1985 and PhD degree from Paris XII University in 1988. After a post-doc position in SAFT-Leclanché Company, Poitiers, France where she developed $\mathrm{Li} /$ Liquid cathode batteries, and several positions at CNRS, she integrated the Bioenergetic and Protein Engineering laboratory, Marseille (France) leading a group focusing on the functional immobilization of redox enzymes on nanostructured electrochemical interfaces. Her aim is to understand the molecular basis for the oriented immobilization of enzymes on electrochemical interfaces favoring fast electron transfer process. She developed original electrochemical interfaces for catalytic reduction of metals by cytochromes, as well as for catalytic transformations of $\mathrm{H}_{2}$ and $\mathrm{O}_{2}$ by hydrogenases and multi copper oxidases respectively. Recently she designed the first high temperature $\mathrm{H}_{2} / \mathrm{O}_{2}$ enzymatic fuel cell. She is currently chair elect of the International Society of Electrochemistry, division Bioelectrochemistry, and member of the bioelectrochemical Society committee.

Dr. Anne de Poulpiquet got interested in bioelectrochemistry during her PhD dedicated to the development of enzymatic $\mathrm{H}_{2} / \mathrm{O}_{2}$ biofuel cells. She obtained her degree under the direction of $\mathrm{Dr}$. Elisabeth Lojou at Aix-Marseille University (France) in 2014. During a postdoctoral stay at the Institute of Molecular Sciences in Bordeaux (France), she studied bipolar electrochemistry and the interplay between electrochemistry and light generation (electrochemiluminescence, fluorogenic electrochemical reactions). She then joined the Bioenergetic and Protein Engineering Laboratory (Marseille, France) as an assistant professor. Her research interests now focus on coupling electrochemistry to optical techniques to study the electro-enzymatic reactivity. 


\section{Glossary}

ADH: alcohol dehydrogenase

AFM: atomic force microscopy

ATR-FTIR: attenuated total reflectance Fourier transform infrared spectroscopy

ATR-SEIRA: attenuated total reflectance surface-enhanced infrared absorption spectroscopy

BOD: bilirubin oxidase

ChOx: cholesterol oxidase

CLSM: confocal laser scanning microscopy

Cp: ceruloplasmin

CcP: Cytochrome $c$ peroxidase

DET: direct electron transfer

DMPC: dimyristoylphosphatidylcholine

DTSP: dithiobis(succinimydil propionate)

$\mathrm{ECL}$ : electrogenerated chemiluminescence

EM-CCD: electron multiplying charge-coupled device

FAD: Flavin adenine dinucleotide

FM: Fluorescence Microscopy

FRET: Förster resonance energy transfer

GC: generation collection

GDH: glucose dehydrogenase

GOx: glucose oxidase

HDMS: hexamethyldisiloxane

HOPG: highly oriented pyrolytic graphite

HRP: horseradish peroxidase

HS-AFM: high-speed atomic force microscopy 
LOx: lactate oxidase

MET: mediated electron transfer

MOF: metal organic framework

NAD+/NADH: nicotinamide adenine dinucleotide

NE: nano-electrode

NiR: nitrate reductase

PEDOT: poly(3,4-ethylenedioxythiophene)

PEI: polyethyleneimine

PM-IRRAS: polarization modulation infrared reflectance absorption spectroscopy

PMT: photomultiplier tube

PMV: protein monolayer voltammetry

PS: polystyrene

PSS: polystyrene sulfonate

QCM: quartz crystal microbalance

SAM: self-assembled monolayer

SECM: scanning electrochemical microscopy

SEM: scanning electron microscopy

SERS: surface-enhanced Raman spectroscopy

SM: single molecule

SPECM: scanning photo-electrochemical microscopy

SPM: scanning probe microscopy

SPR: surface plasmon resonance

STED: stimulated emission-depletion microscopy

STM: scanning tunneling microscopy

TEM: transmission electron microscopy 
UME: ultra-micro electrode

\begin{abstract}
Redox enzymes, that catalyze electron transfer reactions in living organisms, can be used as selective and sensitive bioreceptors in biosensors, or as efficient catalysts in biofuel cells. In these bioelectrochemical devices, the enzymes are immobilized at a conductive surface, the electrode, with which they must be able to exchange electrons. Different physico-chemical methods have been coupled to electrochemistry to characterize the enzyme-modified electrochemical interface. In this review, we summarize most efforts performed to investigate the enzymatic electrodes at the micro and even nanoscale thanks to microscopy techniques. Contrary to electrochemistry, which gives only a global information about all processes occurring at the electrode surface, microscopy offers a spatial resolution. Several techniques have been implemented: mostly scanning probe microscopy like atomic force microscopy (AFM), scanning tunneling microscopy (STM) and scanning electrochemical microscopy (SECM), but also scanning electron microscopy (SEM) and fluorescence microscopy. These studies demonstrate that various information can be obtained thanks to microscopy at different scales. Electrode imaging has been performed to confirm the presence of enzymes, to quantify and localize the biomolecules, but also to evaluate the morphology of immobilized enzymes, their possible conformation changes upon turnover, and their orientation at the electrode surface. Local redox activity has also been imaged and kinetics has been resolved.
\end{abstract}

\title{
Introduction
}

Enzymes are the indispensable catalysts of the plentiful metabolic reactions in living organisms. These complex macromolecules have unequaled properties, notably their high selectivity and turnover rate. Among them, redox enzymes catalyze electron transfer reactions involved in mechanisms such as respiration or fermentation. Electrochemists have inserted them quite early in bio-electrochemical devices like biosensors where they are used as very selective and sensitive bio recognition elements, or bio-fuel cells where they act as powerful catalysts thanks to their high turnover, high specificity, and low overpotentials. ${ }^{[1]}$ Enzyme immobilization at the electrode surface is a critical step in the design and fabrication of these bio-devices. ${ }^{[2]}$ The enzymes must retain their full activity, stability and specificity upon immobilization. Moreover, an effective electron coupling between the protein redox centers and the electrode is a key prerequisite to efficient devices. This latter point is not trivial due to the size of the molecules and their structural complexity. ${ }^{[3]}$ The enzyme activity relies on a redox active site mostly deeply buried in the enzyme dielectric structure, and sometimes accompanied by electronic relays that 
drive electrons between the active site and the surface of the molecule, which results in a huge anisotropy of enzyme electronic properties. The electronic communication between an enzyme and an electrode can be direct ${ }^{[3-4]}$ (i.e. electrons tunnel directly from the enzyme active site or electron relays to the electrode or reciprocally) or indirect (the indirect electron transfer is also called "mediated electron transfer"). ${ }^{[4]}$ In the direct electron transfer (DET) case, an appropriate enzyme orientation is required since tunneling efficiency decreases exponentially with increasing distance between the electron donor and acceptor. In the mediated electron transfer case (MET) a small redox mediator, either diffusing or immobilized, acts as an electron shuttle between the enzyme and the electrode.

Electrochemical methods, which mainly rely on recording current as a function of applied potential, are convenient not only to evaluate the performance of the bio-devices or to characterize the bioelectrodes, but also to study the fundamental properties of the immobilized enzymatic catalysts. Lots of information can be extracted from the electrochemical signal, and allow characterizing the electrode itself, the redox species and electrochemical reaction, as well as transport mechanism and regime. Tuning the overpotential applied to the electrode allows finely tuning the driving force of the electron transfer reaction, and therefore gives access to the thermodynamic and kinetic properties of the biomolecules as well as information about reaction mechanisms. ${ }^{[5]}$ Finally, the same methods give access to current-voltage behavior that fully characterizes a bio-fuel cell, ${ }^{[6]}$ and to sensitivity, precision, and specificity of a biosensor.

Despite the method strength, limiting one's study to pure electrochemical techniques leads to missing information. The first problem is the difficulty to quantify accurately the amount of immobilized enzyme. Moreover, this total quantity represents inactive, active and electroactive enzyme. Even this latter quantity is mostly unknown due to the electrochemical sensibility, which limits the detection of the enzyme non-catalytic signal. Conformational changes due to immobilization or arising during turnover are also unknown, and so are protein aggregation, denaturation or destructuration possibly appearing upon attachment at the electrode surface. Electrochemistry allows only approximating the distribution of enzyme orientation, a crucial problem in the case of DET. Finally, heterogeneity of catalysis at the electrode surface, already reported for abiotic catalysts, ${ }^{[7]}$ cannot be evaluated since the electrochemical response is averaged over the entire electrode surface. Similarly, electrochemistry gives no data about the enzyme distribution at the electrode surface or in the electrode volume when porous electrodes are used.

To get access to these lacking information and better characterize redox enzymes and bioelectrodes, in the past decade, electrochemistry has been more and more coupled to alternative physical methods. 
Among them quartz crystal microbalance $(\mathrm{QCM})^{[8]}$ and surface plasmon resonance $(\mathrm{SPR})^{\left[{ }^{[9]}\right.}$ allow quantifying the immobilized enzyme. QCM with dissipation gives access to some structural information of the immobilized enzyme. ${ }^{[10]}$ Different declinations of infra-red or Raman spectroscopy (PMIRRAS, ATR-FTIR, ATR-SEIRA, SERRS) ${ }^{[9 a, 11]}$ inform about protein structure, orientation, and redox states. Experimenters sometimes conduct two separate experiments, i.e. they analyze their bioelectrode with a physical method prior to/after the electrochemical experiment. ${ }^{[9]}$ More challenging is the direct or "in situ" coupling where the complementary information is recorded directly during the electrochemical experiment. ${ }^{[10]}$

Here we will focus on the most intuitive way to get spatially resolved information about enzymatic electrodes, i.e. their characterization via different kinds of microscopy. We will concentrate on immobilized redox enzymes, putting aside most developments performed with redox proteins like cytochrome, azurin etc. that present no catalytic activity. We will consider mostly cases where electrons are exchanged between the enzyme and the underlying surface (the electrode), either directly or indirectly. In addition, we will describe surfaces that are not, strictly speaking, electrodes especially since in the process of electrode development researchers sometimes transfer their enzymatic system on another surface (glass etc.) to facilitate the microscopical observation. We do not mean to be exhaustive but to give a general overview on the techniques used, to present what kind of information can be extracted, and what are the current limitations. To facilitate the reader's access to information, we also divide each chapter in subsections according to the techniques used, as far as it is possible and seems consistent.

\section{1- Microscopy... and nanoscopy}

In etymological terms, microscopy means "to observe at the micro scale". Modern microscopes allow going much beyond this scale so we can also consider observation at the nanometric scale ("nanoscopy"). We will describe here techniques that allow imaging the whole electrode sample as well as some that allow the visualization of the single immobilized enzyme molecules. Some permit only electrode observation in the dry state, while others allow working in the wet state; and therefore in situ or ex-situ methods are developed. Due to the difference in scale as well as operating conditions, different techniques allow to meet different purposes with most of the time the general aim of studying how the immobilization process or the chemistry and nature of the electrode surface impact the final enzyme coverage and its activity. ${ }^{[8 c]}$ To answer the questions about what happens to enzyme upon immobilization or submission to potential scanning, either spatially resolved information is collected at the electrode scale, or single molecule (SM) observation is performed. The first methodology allows characterizing the localization of enzyme and catalysis, the dimensions of enzyme 
layer, and the enzyme coverage. The second enables resolving the orientation, morphology, mechanism, kinetics of the enzyme at the SM level, thus avoiding approximations due to averaging.

We do not cover here the characterization of electrode structure prior to enzyme immobilization usually carried out with electron microscopy. However, scanning electron microscopy (SEM) is also used to attest the presence of enzymes in the last steps of electrode modification (see following part) so we could not consider the topic without mentioning this technique. Briefly, SEM consists in scanning a focused electron beam over the sample surface. Upon interaction with the sample, the electrons produce secondary electrons, backscattered electrons, and characteristic X-rays. One or several detectors collect these signals to form images. ${ }^{[12]}$ Resolutions achieved with modern SEMs can be as low as $1-20 \mathrm{~nm}^{[13]}$

\section{1- Scanning Probe Microscopy (SPM)}

In our context, the most widely used techniques are the various scanning probe microscopies (SPM): atomic force microscopy (AFM), scanning tunneling microscopy (STM), and scanning electrochemical microscopy (SECM). These techniques are relatively recent since they date back to the 1980's-1990's. In all these different microscopies, an image is obtained by scanning a micro or nano-probe across the sample surface at a short distance from the surface under study. Accurate control of the probe position is achieved via a $X, Y, Z$ piezo positioning system. The scanning probe techniques are non-invasive methods and they can be adapted to make measurements possible under physiological conditions. The nature of the recorded signal is mechanical in case of AFM, electrochemical in case of SECM, and electronic in case of STM. The signal is influenced by the nature and reactivity of the sample, and by the distance between the probe and the sample.

\subsection{1- Scanning Tunneling Microscopy (STM)}

STM was developed by Rohrer and Binnig, ${ }^{[14]}$ who were awarded the 1986 Nobel Prize in physics for this invention. It is all the more noteworthy that it allowed for the first time resolution at the atomic level. STM relies on recording local electronic properties of a sample by imposing a bias voltage between the tip and the scanned sample and recording the resulting tunneling current. Either the scan is performed at a constant distance from the sample and the resulting tunneling current is recorded, or the scan is performed at constant tunneling current and the distance is adapted, which is measured by recording the required voltage at the piezo. Initially developed to visualize surfaces at the atomic level and surface topography, STM was early extended to the manipulation of atoms. ${ }^{[15]}$ Moreover, STM in an aqueous environment was realized as early as $1986,{ }^{[16]}$ which allowed applying the technique to characterization of electrochemical reactions and interfaces. ${ }^{[17]}$ STM contains valuable information about the electronic state of adsorbed molecules, which allows their identification and evaluation of 
their reactivity. It gives access to the molecular energy levels relative to the Fermi level of the sample electrode, which is directly relevant to the electro-activity of the immobilized molecules. It is important to note that STM mapping characterizes molecular tunneling conductivity rather than topographic shape. The measures usually establish either the current/bias voltage relation while the sample electrode is kept at constant potential; or the current/sample electrode overpotential relation at constant bias voltage. ${ }^{[18]}$ The temporal resolution of STM is limited to the order of seconds due to the limited scan rate of the instrument. A second limitation is the need for a conductive sample, imposed by the operating mode, but it is not restrictive in our case where electrode surfaces are studied.

\subsection{2- Atomic Force Microscopy (AFM)}

AFM invention was related in $1986 .{ }^{[19]}$ In this technique, a nanometer tip placed at the end of a vibrating cantilever approaches the sample within few angstroms. The cantilever vibrates due to atomic force interactions between the tip and the sample, which depend on their relative distance. The vibration is visualized with a laser beam and a photodetector. AFM can be operated in two different modes: the contact mode and the tapping mode. ${ }^{[8 c]}$ In the first mode, a continuous contact is established between tip and sample. The second mode is a "dynamic" mode in which the tip oscillates at its resonance frequency and the tip-sample distance is adjusted during scanning so that the amplitude of the oscillation is kept constant. AFM provides morphological and mechanical information at the nanometer level. It can therefore attest the presence of enzymes at an electrode surface, allow to decipher between full or incomplete coverage, between mono- and multilayer, and show surface induced conformational changes of proteins. Here we are interested in AFM as a microscopy technique although it has also been developed as a "force-spectroscopy technique" to characterize mechanical properties of proteins, protein-protein and protein-surface interactions, or to evaluate the strength of attachment of an enzyme to a substrate. Tapping mode has been developed in an aqueous environment, thus allowing characterization of the enzyme electrodes in their working state. Indeed "the biological relevance of dry samples might be questioned" since "imaging under ambient conditions implies the application of higher forces on the protein, which could damage or distort the imaged structures". ${ }^{[20]}$ However AFM can rarely provide information about the catalytic activity of immobilized enzymes, although we present here an example where time resolved AFM has enabled to visualize motions of protein domains during the catalytic cycle (see part 8). The use of AFM for our purposes is mainly limited to perfectly planar surfaces: enzyme molecules are hardly detected in case of roughness. Moreover, possible aberrations are well known. ${ }^{[21]}$ The first, known as tip dilation effects, translates the fact that features smaller than the AFM tip appear as wide as those of the tip. ${ }^{[22]}$ The second, called tip compression effect, is the fact that in tapping mode soft molecules usually appear smaller in height than their real size. 


\subsection{3- Scanning Electrochemical Microscopy (SECM)}

Few tens of reviews as well as comprehensive books have been written on SECM. ${ }^{[23]}$ Engstrom and Liu conducted the first investigations of the electrochemical diffusion layer with the help of a microelectrode, which laid the basis of SECM, in the late 1980 's. ${ }^{[24]}$ The technique was simultaneously developed and formalized by Bard and co-workers between 1986-94. ${ }^{[16 b, 25]}$ Since SECM can work in aqueous buffered solutions and in situations as close as possible to native environments of biomolecules, the suitability for bio-applications was ensured since the beginning. ${ }^{\text {[25d] }}$ It is remarkable that reviews on the use of this technique for the study of enzymes were published as early as 2001. ${ }^{[26]}$ We invite the interested reader to read two very comprehensive and complete recent reviews about the nanoscale SECM as well as about experimental details of the technique. ${ }^{[27]}$ We will here mainly focus on the details necessary to understand what concerns redox biomolecules. Although the definitions can slightly vary, we will call ultramicroelectrodes (UME) electrodes with one dimension smaller than $25 \mu \mathrm{m}$, and nanoelectrodes (NE) those that have at least one dimension smaller than 100 $\mathrm{nm}$. The SECM tip, usually a UME (typically a conductive disk of metal or carbon of 5-25 $\mu \mathrm{m}$ diameter), is a sensitive collector of redox species: the recorded signal is a faradaic current that depends on both the distance to the sample and the sample surface redox activity. Usually, the surface under investigation by SECM is called a substrate, but to avoid any confusion with the molecule that reacts with the enzyme also called a substrate, this term will not be used in this review to indicate the surface. Using a bipotentiostat both tip and surface can be polarized. Traditionally the SECM operation modes can be divided into two main categories: the feedback mode and the generation/collection mode, although several modes appeared more recently like the redox competition mode which is of high interest for the study of enzymes. ${ }^{[27 a]}$

In the feedback mode, the steady-state current I Iip measured at the UME depends on the distance $d$ between the tip and the surface. Feedback mode is characterized by approach curves, i.e. currentdistance curves that represent $I_{T}$ as a function of $L$. $I_{T}$ is the ratio of the tip current ( $I_{\text {tip }}$ ) by the infinite distance current $\left(I_{\text {tip }, \infty}\right), I_{T}=I_{\text {tip }} / I_{\text {tip }, \infty}$, and $L$ is the ratio of the distance tip- surface $d$ by the radius of the electrode $a, L=d / a$. Current-distance behavior at an insulating surface gives negative feedback: the current decreases as the tip approaches the surface because diffusion of the redox active species is hindered. On the contrary, a positive feedback is recorded at a conductive surface: the current increases when the tip approaches the surface because molecules oxidized at the tip can be reduced at the surface (or reciprocally), provided that a convenient polarization is applied both to the tip and the surface (in most cases). Some specificities have to be considered in the case where enzyme activity is imaged. ${ }^{[8 c, 28]}$ In the positive feedback mode, a redox mediator that can be both converted at the electrode and by the enzyme has to be used: it is generally not the enzyme natural co-substrate, but 
an artificial reversible redox mediator allowing better reproducibility and higher sensitivity. The feedback mode requires a diffusion controlled conversion of the mediator at the UME. The main problem of feedback mode on conductive surfaces is a high background current. Moreover, the substrate and product of enzyme reaction must not be converted at the UME, and they must not inhibit the mediator conversion at the UME. An adaptation of feedback mode to the specific case of enzyme reaction can be called "enzyme-mediated feedback mode". ${ }^{[25 c, 25 d, 29]}$ The redox mediator in solution is present at the beginning only in the redox state that does not allow the enzymatic reaction, for example only the reduced state. The tip is polarized at a potential that oxidizes the mediator, and positioning the tip above the enzyme switches on the enzymatic reaction. Therefore a positive feedback due to enzymatic reaction is superimposed to negative feedback due to hampered diffusion of the reduced mediator by the electrode surface. The enzymatic reaction has to be fast enough to be detectable.

The generation/collection (GC) mode can itself be divided into either tip generation/substrate (here, surface studied) collection or substrate generation/tip collection (SG/TC). Studying enzyme reaction relies most of the time on SG/TC: a product of the enzymatic reaction occurring at the surface under study is detected at the tip polarized at an appropriate potential. A current is observed only when the tip is located above an enzymatically active domain. The surface under study can be also polarized but in such a way that what is collected at the tip can only be formed by the enzymatic reaction. GC mode is more sensitive than feedback mode since there is no background current. Indeed, in GC mode the specie detected at the tip is present only if it can be formed at the surface. In the case of the product of an enzymatic reaction, the absence of enzyme results in a zero current. On the contrary, in feedback mode, the specie is originally present in the bulk and changes its oxidation state at the tip. In the presence of enzyme, it is recycled in its former oxidation state by reaction at the surface. This redox cycling therefore enhances the tip current (positive feedback) but the background current is not zero. However overlapping of the diffusion layers from the single enzymes leads to lower lateral resolution. ${ }^{[30]}$

Finally, in the redox competition mode, the tip and the enzyme compete for the same substrate. ${ }^{[31]}$ In these conditions the enzyme reaction is detected because it decreases the current at the tip.

SECM provides morphological data but also information about the (electrochemical) reactivity of a sample. It is therefore adapted for immobilized oxidoreductase enzymes. SECM was used very early to characterize biological molecules: kinetics of electron transfer of glucose oxidase (GOx) immobilized on insulating surfaces were elucidated in the early $1990^{\prime}$ s. ${ }^{[25 c, 25 d]}$ SECM is now also used for the optimization and characterization of sensing platforms. The technique is convenient to image local 
enzyme activity, to allow evaluation of kinetics parameters or visualization of reactant diffusion profiles. ${ }^{[19 a]}$ Since the tip dimensions govern spatial and temporal resolution of SECM, various efforts are devoted to design and prepare nano-electrodes to get sub-micrometric resolution. ${ }^{[27 b, 28]}$ Possible problems with SECM are the correct positioning of the tip UME and large imaging times required for large image frames.

Each technique has its own advantages, for SECM it is its specificity and for STM and AFM it is their nanometric resolution that allows locating individual proteins. The disadvantage in both cases is the restricted scanned area or the long time needed to scan a consequent area. Moreover, a recurrent problem of scanning-probe microscopies is the possible contact between tip and sample that could damage the tip and/or the sample and leads for example to tip-induced desorption upon scanning. ${ }^{[32]}$

\section{2- Fluorescence Microscopy (FM)}

On the contrary, fluorescence microscopy is a non-invasive optical technique in which the sample is observed by setting the focal plane through a display of lenses. Upon excitation of the sample by a light source (most of the time a laser beam), fluorophores are excited and re-emit light upon deexcitation. Although not widely used in bioelectrochemistry, fluorescence microscopy is a method of choice in biological experiments. It is therefore compatible with observation of biological samples and very well described for this kind of applications. Moreover, confocal microscopy allows rebuilding 3Dimages by dividing a sample into optical slices with very low depth field and close focal planes, and stacking the obtained images. The approach is limited by the need for photon-emitting species, which is mainly ensured by labeling, although some molecules of biological interest are self-fluorescent. This is for example the case of the amino-acid tryptophan, or of redox cofactors such as flavin adenine dinucleotide (FAD) in its oxidized state, ${ }^{[33]}$ or nicotinamide adenine dinucleotide in its reduced form (NADH). ${ }^{[34]}$ Moreover, the resolution is limited by diffraction. In situ coupling is still technically complex although it is more and more developing in abiotic context as shown by recent reviews or articles. ${ }^{[35]}$

\begin{tabular}{cccc}
\hline Method (Reference) & \multirow{2}{*}{$\begin{array}{c}\text { Temporal } \\
\text { resolution }\end{array}$} & Lateral dimension & Vertical dimension \\
\cline { 3 - 4 } & & $2-3 \mathrm{~nm}$ & $\sim 0.15 \mathrm{~nm}$ \\
\hline AFM & $<100 \mathrm{~ms}$ & $2-3 \mathrm{~nm}$ & $\sim 0.15 \mathrm{~nm}$ \\
\hline HS-AFM $\left(^{[36]}\right)$ & $\sim \mathrm{s}$ & $\sim 100 \mathrm{~nm}$ & \\
\hline SECM $\left(^{[27 b, 37]}\right)$ & $10-1000 \mathrm{~ms}$ & Atomic scale & Atomic scale \\
\hline STM $\left(^{[38]}\right)$ & $\mathrm{ns}$ & Atomic scale & Atomic scale \\
\hline Ultrafast STM $\left.{ }^{[38]}\right)$ & & $\sim 10 \mathrm{~nm}$ & \\
\hline SEM $\left.{ }^{[13]}\right)$ & & & \\
\hline
\end{tabular}




\begin{tabular}{cccc}
\hline Ultrafast SEM $\left({ }^{[13]}\right)$ & a few ps & $\sim 10 \mathrm{~nm}$ & $600 \mathrm{~nm}$ \\
\hline Wide-field FM $\left({ }^{[39]}\right)$ & $\sim 100 \mathrm{~ms}$ & $\sim 250 \mathrm{~nm}$ & 600 \\
\hline Confocal FM $\left({ }^{[39]}\right)$ & $\sim 100 \mathrm{~ms}$ & $\sim 180 \mathrm{~nm}$ \\
\hline Super-resolution FM & $\sim \mathrm{s}$ & $20-30 \mathrm{~nm}$ & \\
(example of STED) $\left.{ }^{[40]}\right)$ & & &
\end{tabular}

Table 1- Spatial and temporal resolutions of the different microscopy techniques. STED stands for Stimulated Emission Depletion.

\section{2-Electrode imaging to confirm the presence of enzymes}

The first justification of the use of microscopy is to prove that the enzyme has been attached at the electrode surface or in the electrode volume. It is not rare to find articles where microscopy has been used to attest the presence of the enzyme molecule after incubation of the electrode in the enzyme solution, or after deposition of the enzyme solution on top of the electrode. This could be considered as a first step towards quantification of enzyme, or localization of its distribution, although these observations are not precise enough so far.

\section{1- Electron Microscopy}

Various articles report the use of SEM to image the electrode surface after enzyme immobilization. We will mention here only a few recent studies. This technique is very interesting since observations are possible down to a very low scale. The presence of laccase on ferromagnetic nanoparticles bound by

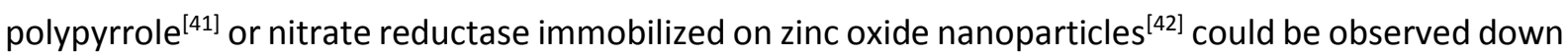
to very small scales: features as small as few tens of nanometers could be easily distinguished. The surface or nanostructure are compared prior to and after enzyme addition and modifications indicate a successful enzyme immobilization. In one study, not only the presence of enzyme but also the influence of enzyme solution on structure preservation has been investigated. After immobilization of GOx on carbon fibers, these latter kept their structure but deposition of the enzyme solution on the materials was more or less homogeneous depending on their pretreatment, likely due to differences in wettability. ${ }^{[43]}$ In most cases, although a change of morphology or texture of the surface is detected upon enzyme addition, no more information is obtained. Several causes can explain this. First, the enzyme protein structure is dielectric, therefore leading only to a low-contrast in the absence of metallization. The complexity of most enzyme solutions, that contain additives due to the purification (like glycerol, buffer, salts, etc.) result in a paste where enzyme remains indistinguishable. In the case of micro- or nanostructured electrodes, nothing indicates if the enzyme solution has penetrated inside the 3D structure or if it has only covered the surface. For example, a well-defined nanostructure was formed by electrodeposition of poly(3,4-ethylenedioxythiophene) (PEDOT)-graphene oxide 
nanosheets on the electrode surface. The highly porous composite film comported well-dispersed pores and its high surface area provided a proper matrix for enzyme immobilization. After entrapping laccase, the pores were filled with enzyme and the porous structure was not much visible. This indicates a successful enzyme immobilization, but also raises the question of whether pores may be closed. ${ }^{[44]}$ Similarly, the nanostructure formed by vertically aligned CNTs on a composite aluminium foil/polymer was erased by addition of $\mathrm{GOx} \cdot{ }^{[45]}$ Magnetic $\mathrm{Fe}_{3} \mathrm{O}_{4}$ nanoparticles covered with carboxymethylated chitosan could be individually resolved, and laccase addition resulted in much bigger clusters. ${ }^{[46]}$ In another study, PEDOT-microspheres were decorated with platinum nanoparticles clearly visible by SEM. Upon GOx addition, a blurry surface with less visible nanoparticles was observed. ${ }^{[47]}$ Upon urease immobilization on graphene oxide sheets, the sheet-like structure became more "regular" and features like aggregates or bundles appeared. ${ }^{[48]}$ When horseradish peroxidase (HRP) was immobilized on a network of polyaniline nanotubes grown on glassy carbon, the surface appeared more "compact and undulant". ${ }^{[49]}$ In the same article, the use of transmission electron microscopy is worth mentioning since patterns corresponding to HRP could clearly be distinguished on the nanotubes (Figure 1). The presence of individual enzymes on nanotubular nucleoproteins could also be visualized by SEM. ${ }^{[50]}$

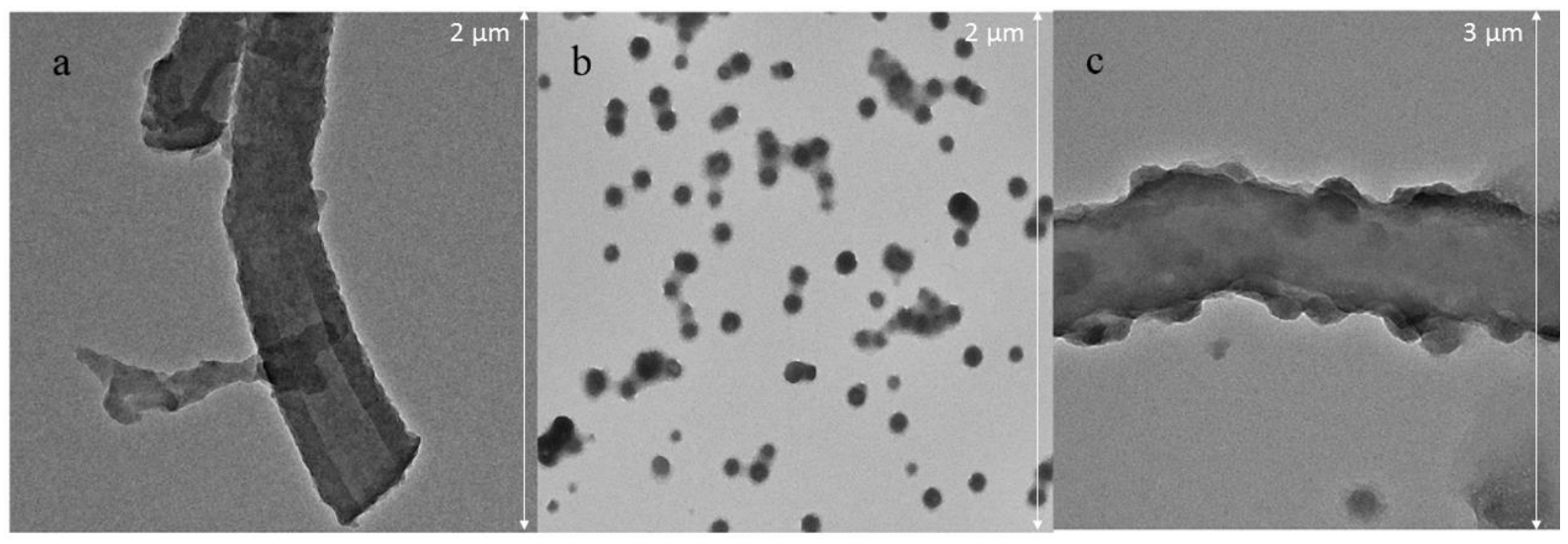

Figure 1 - TEM Images of: a- Polyaniline nanotubes (PANT); b- Horseradish peroxidase (HRP) and c- HRP immobilized at PANT. Reproduced with permission from reference ${ }^{[49]}$

\section{2- $A F M$}

Redox active biomolecules (cytochrome $b_{562}$ ) covalently attached on carbon nanotubes have also been visualized by AFM. ${ }^{[51]}$ To the best of our knowledge, this has not been realized so far for enzymes. This latter technique can provide much more information about enzyme coverage, thickness of the enzyme layer and even visualization of single enzyme molecules. However, in this part, we mention only examples where morphological changes of the electrode surface induced by enzyme attachment were visualized. The presence of Cholesterol Oxidase (ChOx) deposited by Langmuir-Blodgett technique on 
a carbon electrode was attested by AFM imaging, that evidenced a roughness decrease upon formation of ChOx films. ${ }^{[52]}$ In the case of layer-by-layer assembly of nanographene / GOx / Chitosan alternated with polystyrene sulfonate (PSS) on gold modified with PEDOT, SEM and AFM were associated to determine the surface characteristics of the layers and their thickness. ${ }^{[53]}$ AFM imaging after immunolabelling also attested the adsorption of horseradish peroxidase (HRP) on a gold (111) surface decorated with a phospholipid bilayer, consistently with negatively charged phospholipids and positively charged HRP at the experimental pH (6.5). A primary antibody allowed enzyme recognition, while the secondary antibody bore nanoparticles clearly visible as white dots on the AFM image (Figure 2). ${ }^{[54]}$ When HRP was immobilized via 3 different methods (adsorption, cross-linking, covalent binding) on bare or modified polystyrene (PS), AFM showed a clear change in surface morphology upon enzyme addition. Moreover, it proved that enzyme homogeneity, distribution or aggregation depend on the immobilization method. ${ }^{[55]}$ AFM was also combined to fluorescence microscopy imaging to characterize a laccase cross-linked via glutaraldehyde in a polythiophene-based conducting polymer. A decrease in surface roughness was evidenced by AFM upon enzyme coating, while the laccase molecules appeared as spots in the self-fluorescent polymer ${ }^{[56]}$ AFM requires mostly planar surfaces, however it also allowed to study the presence of laccase on highly-oriented pyrolytic graphite (HOPG) after different adsorption times $(20 \mathrm{~s}, 30 \mathrm{~min}$ and $24 \mathrm{~h}) \cdot{ }^{[57]}$ We quote a latter case where individual molecules were not distinguished: AFM was used to show changes of surface morphology at the different steps of the creation of an electrode made of screen printed carbon covered by chitosan and finally by glutathione-S-transferase. ${ }^{[58]}$
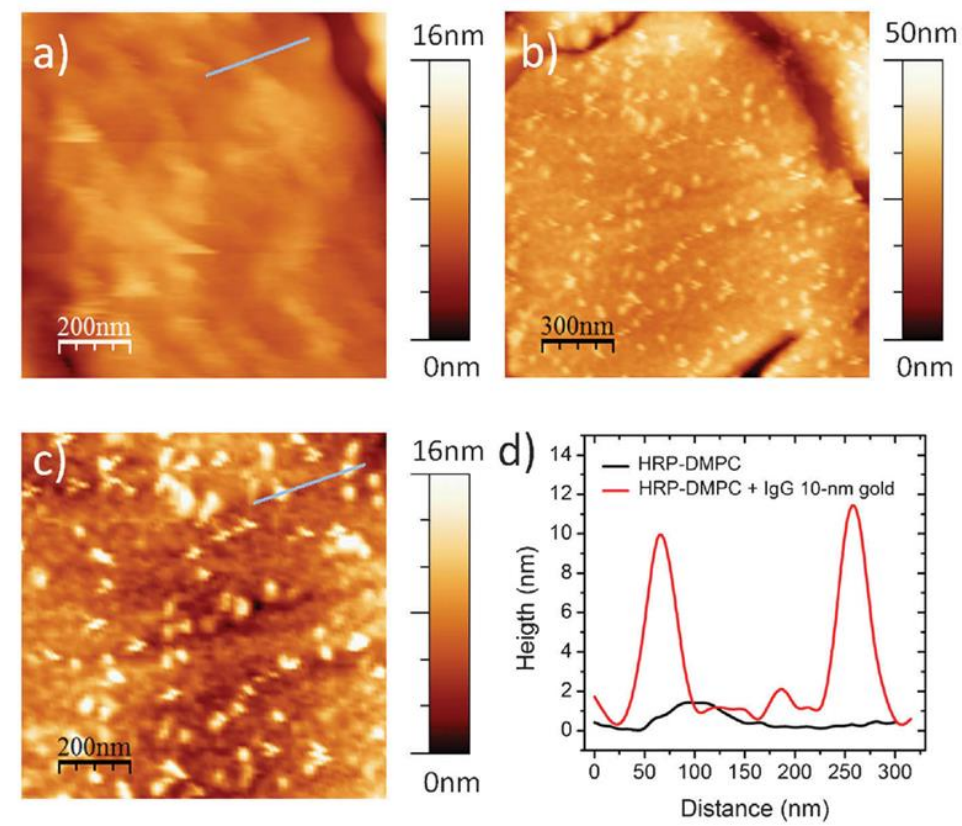

Figure 2 - Contact mode AFM image of a bilayer of horseradish peroxidase (HRP) and dimyristoylphosphatidylcholine (DMPC) : a- before and b- after labeling with antibody IgG 10-nm gold 
conjugate; c- Magnification of a specific area of (b) and d- line-scan profiles indicated in (a) and (c). Reproduced with permission from reference ${ }^{[54]}$

\section{3- Other techniques}

With this aim of attesting the presence of enzymes, other techniques are more anecdotic. STM was combined with ATR-SEIRA to study the immobilization of laccase on modified gold electrodes. ${ }^{[59]}$ The aim of microscopy was to provide information about the changes in topography and structure of the electrode surface associated with chemical modification of the electrode and with enzyme attachment. Two different surface modifications were conducted and the enzyme was covalently attached via amide bond formation. In both cases, protrusions attributed to single enzyme units were observed, although their height was much lower than expected. The enzyme coverage could not be evaluated but the two different surface modifications were compared. The higher coverage could be explained by the bigger amount of functional groups available for covalent bond formation. SECM also allows detecting or localizing enzymes, but most of the time this detection relies on the enzyme activity so that only active immobilized enzymes are mapped or detected. ${ }^{[60]}$ We will describe this in more details in following parts. Not only SPM or electron microscopy have been used. Catalase molecules covalently attached at magnetic beads were evidenced by electrochemiluminescence (ECL) whose advantage is resolution due to confinement of the reaction at the electrode surface. A magnet was placed under the working electrode chip, divided into 73 microchambers, to attract the magnetic beads at the surface of the electrode. $\mathrm{H}_{2} \mathrm{O}_{2}$, generated in situ by incomplete $\mathrm{O}_{2}$ reduction at the working electrode surface, served both as the enzyme substrate and as a coreactant for ECL with luminol as a luminophore. Rapid consumption of $\mathrm{H}_{2} \mathrm{O}_{2}$ by catalase results in $\mathrm{ECL}$ quenching, which allowed sensitive detection of the enzyme down to concentrations as low as $90 \mathrm{fM} .^{\left[{ }^{[1]}\right.} \mathrm{ECL}$ was recorded with an image intensifier and an electron multiplying charge coupled device (EM-CCD) camera mounted on top of an upright microscope. The presence of enzymes in polymer membranes was also attested by confocal Raman microscopy. Raman spectrum allows determining the composition of a sample; and a fixed sample volume is observed by using confocal microscopy. A laccase air-breathing cathode was constructed by encapsulating a laccase in a modified Aquivion polymer deposited on a graphite felt. Spectral features associated with the enzyme, the amide I band at $1673 \mathrm{~cm}^{-1}$ and features associated with vibrations of functional groups near the enzyme active site, were evidenced. This technique could enable in the future both quantification of the enzyme and studies of its active site with a spatial resolution approaching the diffraction limit of the excitation beam. ${ }^{[62]}$ Finally, we noted one example using fluorescence microscopy. After introducing metalorganic frameworks (MOF) in an aqueous solution of HRP labeled with fluorescein isothiocyanate under agitation, the presence of the enzyme in MOFs was attested by confocal fluorescence 
microscopy. The respective dimensions of enzyme and MOF cavity are $4 \times 4.4 \times 6.8 \mathrm{~nm}^{3}$ and $5.5 \mathrm{~nm}$ in diameter. Confocal image of a crystal of MOF (around $2 \mu \mathrm{m}$ ) showed an even distribution of enzyme throughout the crystal, ${ }^{[63]}$ which was furthermore confirmed by SEM-EDS images showing even distribution of sulfur in the crystal (Figure 3). This characterization was performed before immobilization of the enzyme-loaded MOF on a glassy carbon electrode, on which MET was established with hydroquinone as a redox mediator.

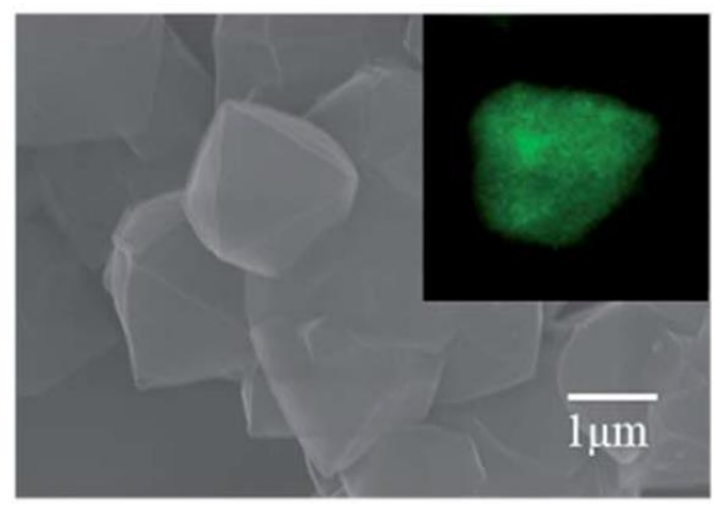

Figure 3- SEM image of the metal-organic framework PCN-333(Fe). Inset: confocal microscopy image of the PCN-333(Fe) after encapsulation of labeled HRP. Reproduced with permission from reference [63]

\section{3- Characterization of the enzyme coverage}

A recurrent problem in enzyme electrochemistry is the difficulty to quantify the amount of immobilized/active/electroactive enzyme. Enzyme coverage is however expected to play a key role in enzymatic activity, simply considering that in vivo enzymatic reactions take place in crowded molecular environments. ${ }^{[64]}$ In the case of direct electron transfer, quantification of electroactive enzymes would be possible by the integration of the non-catalytic signal observed in the absence of substrate. However, this latter is rarely detected. For example, only two publications relate its observation for the enzyme hydrogenase. ${ }^{[6,65]}$ Therefore, authors most of the time consider that enzyme quantity is below the electrochemical detection limit. QCM and SPR are appropriate tools to determine the mass adsorbed at the electrode surface. The coverage can also be calculated or at least approximated thanks to microscopy techniques, by counting the number of molecules per unit of surface area of the electrode as soon as individual molecules can be distinguished. This was realized as early as 1998 by AFM that showed individual molecules of catalase physisorbed or covalently attached on gold modified by self-assembled monolayers (SAMs) of thiols bearing three different chemical end functions. As expected, the coverage extent was greatly influenced by the SAM nature and bond, varying between $38 \%$ for physical adsorption on short chain carboxyl-terminated SAMs and $100 \%$ for covalent bonding on mixed SAMs. ${ }^{[66]}$ If it is not always possible to count the molecules, at least observing the interval 
between molecules or the presence of voids indicates if full layers are formed. Myrothecium verrucaria bilirubin oxidase (BOD) and Trametes hirsuta laccase were adsorbed on bare gold surfaces from enzyme solutions at different concentrations, and the samples were studied by AFM in liquid cell. Au grains were observable by AFM and a homogeneous and full coverage was obtained upon enzyme adsorption from concentrated solutions. Smaller concentrations led to sub-monolayers. AFM would therefore enable the measurement of enzyme adsorption isotherms on the electrode surface. Also, no desorption was observed over $1 \mathrm{~h}$, confirming the irreversible character of adsorption on gold. ${ }^{[67]}$ The adsorption of lactate oxidase on glassy carbon or highly oriented pyrolytic graphite (HOPG) was also characterized by AFM: at short adsorption time incomplete monolayers where enzymes aggregate laterally were formed, so that full monolayers were observed for longer adsorption times (Figure 4). ${ }^{[20]}$
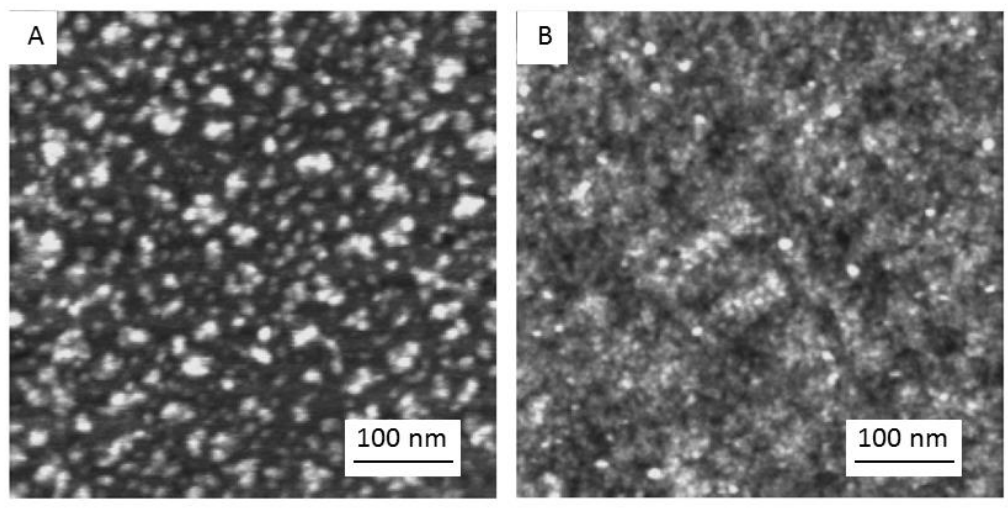

Figure 4- AFM images in tapping mode in buffer of lactate oxidase adsorbed on HOPG. A- Incomplete enzyme layer. B- Full enzyme layer. Reproduced with permission from reference ${ }^{[20]}$

Determining the height of the protein layer can also tell if enzyme monolayer or multilayers are formed. The presence of a monolayer of Aquifex aeolicus membrane-bound hydrogenase on an electrode modified with hydrophobic SAM was for example revealed by AFM. ${ }^{[11 a]}$ A similar indication was obtained with another globular enzyme, Ralstonia eutropha membrane-bound hydrogenase, immobilized on a gold electrode. ${ }^{[68]}$ Trametes versicolor laccase was immobilized on gold following three different strategies: adsorption, covalent attachment, encapsulation in a three-dimensional solgel matrix. AFM allowed saying if enzymes formed monomers or aggregates (thanks to lateral resolution of the spots), if enzyme layers were compact, and if monolayers were formed. ${ }^{\left[{ }^{[9]}\right.}$ Adsorbed enzymes formed a compact layer on top of which SM or aggregates accumulated (height values $>10$ $\mathrm{nm})$. More or less the same was observed upon covalent binding, except that the aggregates seemed to concentrate at the grain boundaries. Sol gel built a 20-75 nm-thick matrix in which laccase molecules were stacked at different depths. Therefore, much more molecules were immobilized with this latter strategy. ${ }^{[69]}$ Homogeneity, distribution or aggregation was also shown by AFM to depend on immobilization method for HRP bound on polysaccharide via three different methods (adsorption, 
cross-linking, covalent binding). ${ }^{[55]}$ After deposition of nitric oxide synthase oxygenase solution at $\mathrm{pH} 7$ or 8.6 on positively charged polyethyleneimine (PEI) on HOPG, AFM imaging in ambient conditions showed more enzymatic clusters at higher $\mathrm{pH}$. This is consistent with an electrostatically driven immobilization of the enzyme, which is more negatively charged at higher $\mathrm{pH} .{ }^{[70]} \mathrm{AFM}$ was also coupled to electrochemistry to reveal the impact of spatial organization at the nanometer scale to enzymatic activity of GOx immobilized on bacteriophage virus used as a platform on gold electrodes. ${ }^{[71]}$

Surface coverage can also be deduced from STM images, as was done for Achromobacter xylosoxidans nitrite reductase $(A x \mathrm{NiR})^{[72]}$ or Escherichia coli cytochrome c nitrite reductase (Ec cyt.c NiR). ${ }^{[73]}$ In situ STM image of Ec cyt.c NiR immobilized on Au (111) showed that the density of enzymes was below the monolayer coverage: $0.5 \pm 0.1 \mathrm{pmol} . \mathrm{cm}^{-2}$, which agreed with the fact that it could not be detected in cyclic voltammetry. This coverage estimation enabled further calculation of kinetic constants. ${ }^{[73]}$ STM images also allowed estimating coverages of $E$. coli copper efflux oxidase on a gold electrode, and showing that they depend on the SAM chemistry. ${ }^{[74]}$

\section{4- Enzyme localization}

Studying the distribution of enzyme localization is important to help fundamental understanding and characterization of bioelectrochemical devices. It can also give clues about the efficiency of the modification method for the biomolecules. If not all enzymes are submitted to the same (and optimal) environment, they are not likely to be able to all work in optimum conditions. Not only are the electrode materials presumably heterogeneous, but enzyme deposition methods can lead to heterogeneities. It is important to understand where enzymes preferentially immobilize on planar electrodes: is it on the well-defined surface or rather on defects? If a drop of enzyme solution is deposited at the electrode surface and left to dry, concentration of enzyme should not be the same at the center and peripheries due to equilibrium forces. It is also difficult to realize homogeneous chemical modifications of electrodes. Are enzymes preferentially immobilized where the modification is effective or not? The problem becomes even more complex when tridimensional electrodes are used and several questions arise. Does the enzyme stay on the surface of the 3D network or does it penetrate the pores? Is the enzyme distribution in the volume sample homogeneous or is there a gradient of enzyme concentration inside the pores? Could the enzymes clog the pores, as already mentioned?

\section{1- Scanning Probe Microscopies}

Here again SPM can be used, however it is quite rarely reported due to the long times required to scan the entire surface of an electrode. The following case is interesting although it does not deal with enzymes. Mapping of redox marked proteins on conductive surfaces was realized by "molecular 
touching AFM-SECM". The AFM-SECM probe allows combining force sensing by AFM in tapping mode, and SECM for the electrochemical detection or redox-tagged immobilized biomacromolecules. Regular patterns of redox-marked protein domains were drawn on the surface and accordingly imaged. The technique associates AFM high resolution and SECM selectivity, and allows discriminating between the redox marked proteins and inactive spots with similar dimensions. The resolution was in the $100 \mathrm{~nm}$ range, and sensitivity in the $10^{-12} \mathrm{~mol} . \mathrm{cm}^{-2}$ protein coverage. ${ }^{[75]}$ Some examples of enzymatic electrode studies are reported. Laccase was covalently immobilized via amide or amine bonds on amorphous carbon nitride. In the case of amide bond, three different zones were observed: bare carbon nitride; enzyme layer; and zones in-between that were attributed to deformed enzymes. The attribution was realized thanks to the correlation between AFM topography image and estimation of height profile via nanoscratching. In the case of amine bond, a full layer of enzymes was formed. ${ }^{[76]}$ An Os-polymer film decorated with gold nanoparticles was imaged by AFM prior to and after incubation in a GOx solution. A clear change in the morphology of the film was observed and GOx seemed to predominantly adsorb on AuNPs rather than on polymeric domains. This explained why a higher activity was obtained compared to polymer alone, where an even distribution of the enzyme was observed. ${ }^{[77]}$ Finally, the evolution of localization of poly(3-hydroxy-butyrate)depolymerase on poly-L-lactic thin films during adsorption was imaged over time with time-resolved AFM. The enzyme was shown to first gradually cover the surface, and then to form a 2-D network with molecules attaching laterally to each other. ${ }^{[78]}$

\section{2- Fluorescence Microscopy}

Fluorescence microscopy has also proved useful although its use remains quite rare. Distribution of a labeled enzyme in different polymer matrices ${ }^{[79]}$ or in carbon fibers ${ }^{[80]}$ was studied using confocal laser scanning fluorescence microscopy (CLSM). CLSM allows rebuilding three-dimensional images of optically transparent samples by stacking planar images recorded at different focal planes. In the case of transparent polymer matrices, it was therefore a non-invasive method: 3D images of the $80 \mu \mathrm{m}$ thick films were realized. However only dried scaffolds were imaged. ${ }^{[79 b]}$ In the second case, 2Dsections of the cut carbon fiber were imaged. ${ }^{[75]}$ Alcohol dehydrogenase, ${ }^{\left[{ }^{[79 a]}\right.}$ malate dehydrogenase alone ${ }^{[79 b]}$ or in cascade with citrate synthase and aconitase ${ }^{[81]}$ were immobilized in 3D macroporous polymeric scaffolds ${ }^{[79,81]}$ based on polysulfonic polymers or chitosan and their hydrophobic derivatives. Staining the polymer and labelling the enzyme with different fluorophores allowed visualizing both the polymer structure and the enzyme distribution in the matrix. Interestingly, it was shown that enzyme distribution was strongly influenced by the polymer charge ${ }^{[79 a]}$ or hydrophobicity ${ }^{[79 b, 81]}$ but no influence of the enzyme tag was detected whatever its charge, suggesting that labeled enzymes behaved as unlabeled ones. Finally, in case of enzyme cascades, evaluation of enzyme distribution, co-localization and self-aggregation could be performed by exciting separately the different fluorophores. The 
distance between the different fluorophores, thus the degree of aggregation induced upon immobilization in polymer scaffolds, was determined by Förster resonance energy transfer (FRET). ${ }^{\text {[81- }}$ ${ }^{82]}$ The use of fluorescence microscopy to characterize enzyme encapsulated in polymeric 3D scaffolds showed that, while it is commonly accepted that the enzyme is homogeneously distributed in the polymer matrix, it more often forms aggregates localized in discrete regions (channels...). The method finally allowed selecting the polymer that creates a better enzymatic system. ${ }^{[79 b, 81-82]}$ Fluorescence microscopy was also used to image a cross section of a carbon fiber electrode. Labelled BOD had been imbricated in the fiber during the fabrication process by wet-spinning a mixture of CNTs and enzyme. CLSM proved that enzyme was indeed present and quite homogeneously distributed in the core of the fiber (Figure 5). ${ }^{[80]}$

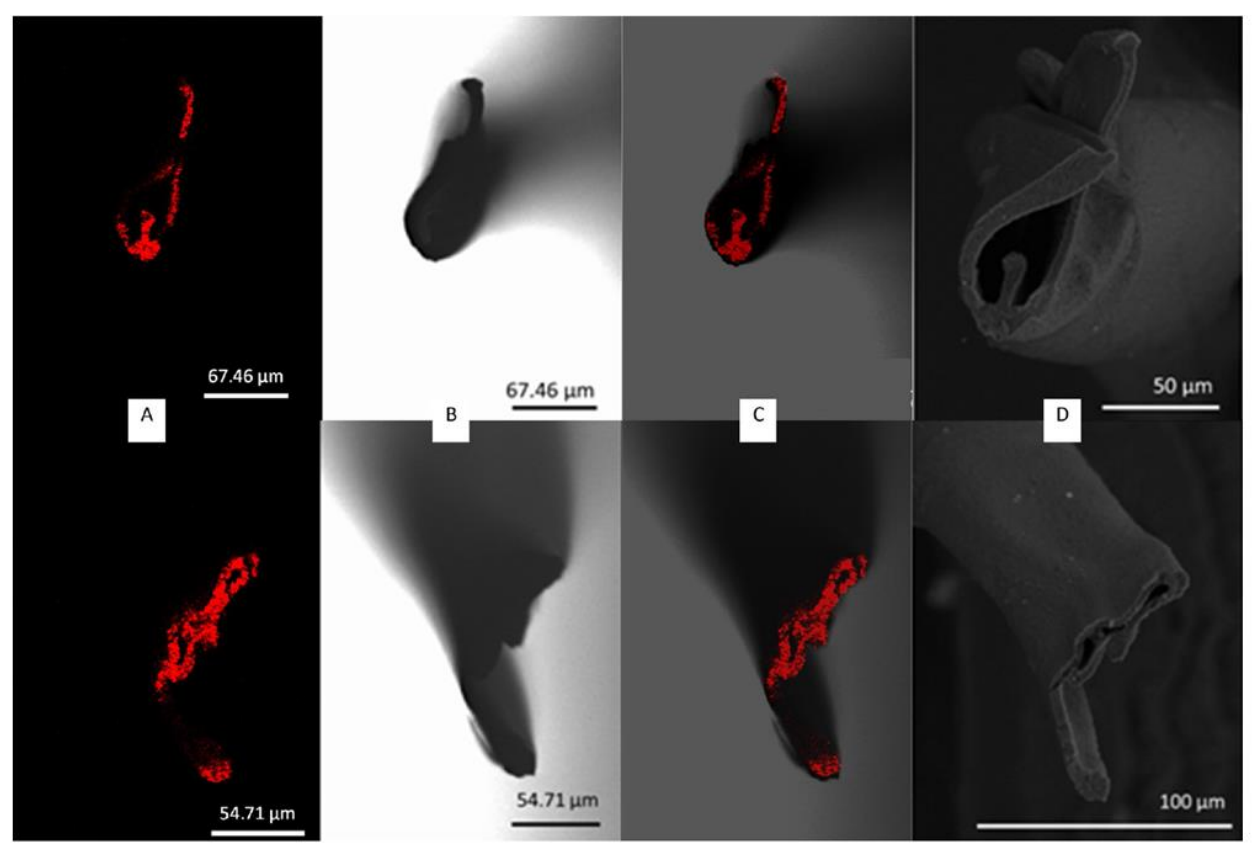

Figure 5- Images of two cross sections of a carbon fiber electrode containing a labelled enzyme to evidence localization of the enzyme in the core of the electrode. Pictures obtained by A-confocal laser scanning microscopy; B- optical microscopy and D- Scanning electron microscopy. C- shows the superposition of $A-$ and $D$. Reproduced with permission from reference ${ }^{[80]}$

The use of fluorescence to localize enzyme requires the presence of photon-emitting species in the enzyme structure. A possibility is to image the endogenous fluorescence of an amino-acid residue (tryptophan is well-known to emit between $300-350 \mathrm{~nm}$ ) or of a cofactor (such as flavin cofactors). To the best of our knowledge, this has never been reported to this purpose. Therefore it is necessary to tag the enzyme. In the reported studies, a fluorescent label was covalently linked to the enzyme. It must be noted however that the tag efficiency remained quite poor. ${ }^{[80-81]}$ Another possibility is to 
merge the protein of interest with a fluorescent protein such as the green fluorescent protein (GFP) and similar proteins. This has proved efficient for in vivo localization of proteins ${ }^{[83]}$ but we might expect that purification of the merged complex would be complicated. Indeed enzymes of interest have molecular weight around 50-100 kDa which makes a $30 \mathrm{kDa}$ tag in the shape of a barrel of beta sheets not negligible. Moreover, it is highly probable that in that case the labeled enzyme would not behave as the unlabeled one. To conclude, this method is extremely interesting since it is one of the rare nondestructive ways to study enzyme immobilization in a 3D matrix. However, it is constraining in that the matrix needs to be optically transparent.

\section{5- Morphology of immobilized enzymes}

Conformational distortion upon enzyme attachment may induce loss of activity, so it is important to correlate adsorption protocols to changes in morphology and biological activity. SM observation of proteins also allows characterizing if enzymes possibly change shape/flatten upon immobilization at the electrode surface, by comparing dimensions given by X-Ray crystallography and dimensions observed by microscopy.

\section{1- STM}

Description at the molecular level of $E$. coli cyt.c nitrite reductase, a decaheme containing homodimer that reduces nitrite into ammonium, immobilized on monocrystalline gold was performed by STM. Without nitrite, $10 \mathrm{~nm}$-wide regions with 5-15 nm lateral dimensions were distinguished, which agreed well with crystallographic data, although a little bit bigger. The authors explained that this observation, which is quite common, arose either from water layers surrounding the protein or from tip convolution. Interestingly, in the presence of the nitrite substrate, the molecules appeared smaller: the enzyme structure was more compact. ${ }^{[73]}$

\section{2- $A F M$}

AFM in tapping mode under liquid conditions allowed comparing the morphological characteristics of a lactate oxidase enzyme adsorbed on carbon electrodes in full or incomplete monolayers. In incomplete monolayer on HOPG, enzymes were seen both as isolated and laterally aggregated molecules whose height was approximately 3.5-4.5 nm. In full monolayer on HOPG and glassy carbon, the protein lateral dimension was 5-8 nm, while the layer thickness was estimated between 4-6 nm, thus a little bit higher than in incomplete layers. The tip-induced deformation during AFM imaging was considered negligible. ${ }^{[20]}$ Similarly, spherical features approximately $3 \mathrm{~nm}$-high were observed when Aquifex aeolicus membrane-bound hydrogenase immobilized on hydrophobic SAM was examined with AFM. ${ }^{[11 a]}$ AFM also showed that Trametes versicolor Laccase forms upon adsorption a compact layer of 
globular structures with characteristic lateral size in the $5-8 \mathrm{~nm}$ range. ${ }^{[69]} \mathrm{A}$ small enough tip is necessary to get consistent dimensions and thus conclude on the morphology: it was for example 2 $\mathrm{nm}$ in the above-mentioned study. ${ }^{[69]}$ The morphology of Myrothecium verrucaria BOD and Trametes hirsuta laccase adsorbed on bare gold surfaces was studied by AFM in liquid cell. For both enzymes, globular features around $20 \mathrm{~nm}$ in lateral dimension were observed, which is much bigger than the expected 4-6 nm. This was interpreted as aberrations due to size of the tip, which was $20 \mathrm{~nm}$ in diameter. The height was also smaller than expected, which was attributed to tip compression effect. No conclusion about the size was possible with AFM alone, but in conjugation with ellipsometry, electrochemistry and enzymatic assays it suggested a flattening of enzyme on gold surface upon adsorption. ${ }^{[67]}$ In the study already mentioned above where a laccase was immobilized on amorphous carbon nitride, correlation between AFM topography image and estimation of height profile via nanoscratching allowed detecting deformed enzymes standing flat on the surface. ${ }^{[76]}$

\section{6- Enzyme orientation at the electrode surface}

We already described the contribution of microscopy to study enzyme orientation in a previous review. ${ }^{[3]}$ Therefore, we will here only briefly summarize the main articles. Moreover, since we had left over some references in our previous work, we will take the opportunity to introduce them here. Orientation is a major issue when trying to establish direct electronic connection between an enzyme and an electrode, due to the enzyme large molecular size and the anisotropy of its electronic properties. This latter results from heterogeneous distribution of redox active sites (catalytic active site or electron relays) in the protein structure. Marcus theory, which states that the rate of electron transfer depends on the distance between electron donor and acceptor, can be adapted to the special case of enzymes immobilized on an electrode. Usually, a distribution of molecule orientations on the surface results in a distribution of electron transfer rates for electronically addressable enzymes. ${ }^{[84]}$ However if enzymes are randomly orientated, and if we consider a maximum tunneling distance of 14 $\AA_{,}^{[85]}$ the large majority of immobilized enzymes should not be able to directly exchange electrons with the electrode. Therefore, various strategies have been developed to force the enzyme to adopt a specific orientation. The electrode surface can be chemically modified to mimic the protein natural environment, its physiological partner or substrate. Chemical modifications can also induce electrostatic interaction that fit with the enzyme dipole moment. Specific covalent binding can lead to preferential orientation. It is important to check the efficiency of these rational methodologies. A first approach based on pure electrochemistry consists in comparing the ratios of DET and MET obtained with a mediator. Further information can be extracted from the electrochemical signal by modeling to determine the $\beta$ factor that gives clues about the distribution of orientations. ${ }^{[86]}$ Surface infra-red spectroscopy methods like ATR-FTIR, PM-IRRAS are also interesting tools because different 
orientations of enzymes result in different ratios of amide I/amide II bands in the spectrum so that a mean orientation can be extracted from the spectrum. ${ }^{[11 a, 76,87]}$

Microscopy also offers some possibilities. Orientation can be determined by looking closely at individual molecules thanks to single-molecule microscopy, or by measuring height profiles of an enzyme monolayer.

\section{1- $A F M$}

Studying protein orientation with AFM is theoretically possible either directly or indirectly. Direct measure of the height profile of the protein layer by AFM gives information about orientation only if one dimension of the enzyme is larger than the other ones, and is therefore not a valuable method for globular enzymes. An indirect way is to use antibodies that bind to a specific region of the protein. Measuring the volume of the protein / protein-complex, or the height profile of the protein layer, allow to decipher if the antibody is present and therefore to deduct the orientation of the protein. ${ }^{[88]}$

In the case of enzymes, we could only find articles relating to the first strategy, and therefore only enzymes with specific shapes are concerned. AFM tapping mode in air was used to study the orientation of GOx, an elliptic enzyme with a small axis of around 6-8 $\mathrm{nm}$ and a long axis about 10-14 $\mathrm{nm}$, on plasma polymerized thin films of hexamethyldisiloxane (HMDS) with different surface properties. When HDMS was hydrophobic and neutral, an incomplete enzyme coverage was recorded together with a protein height of 9-14 $\mathrm{nm}$. This indicated that GOx was perpendicular to the surface, consistently with the interaction between the surface and GOx hydrophobic domain (Figure 6). For hydrophilic and positively charged HDMS, a compact layer with GOx disposed parallel to the surface was observed (Figure 6). The same orientation could be recorded for hydrophilic and negatively charged polymers, with however isolated molecules. The two latter cases are consistent with the creation of electrostatic interactions, since the enzyme $\mathrm{pl}$ is 4.2. ${ }^{\left[{ }^{89]}\right.}$ 


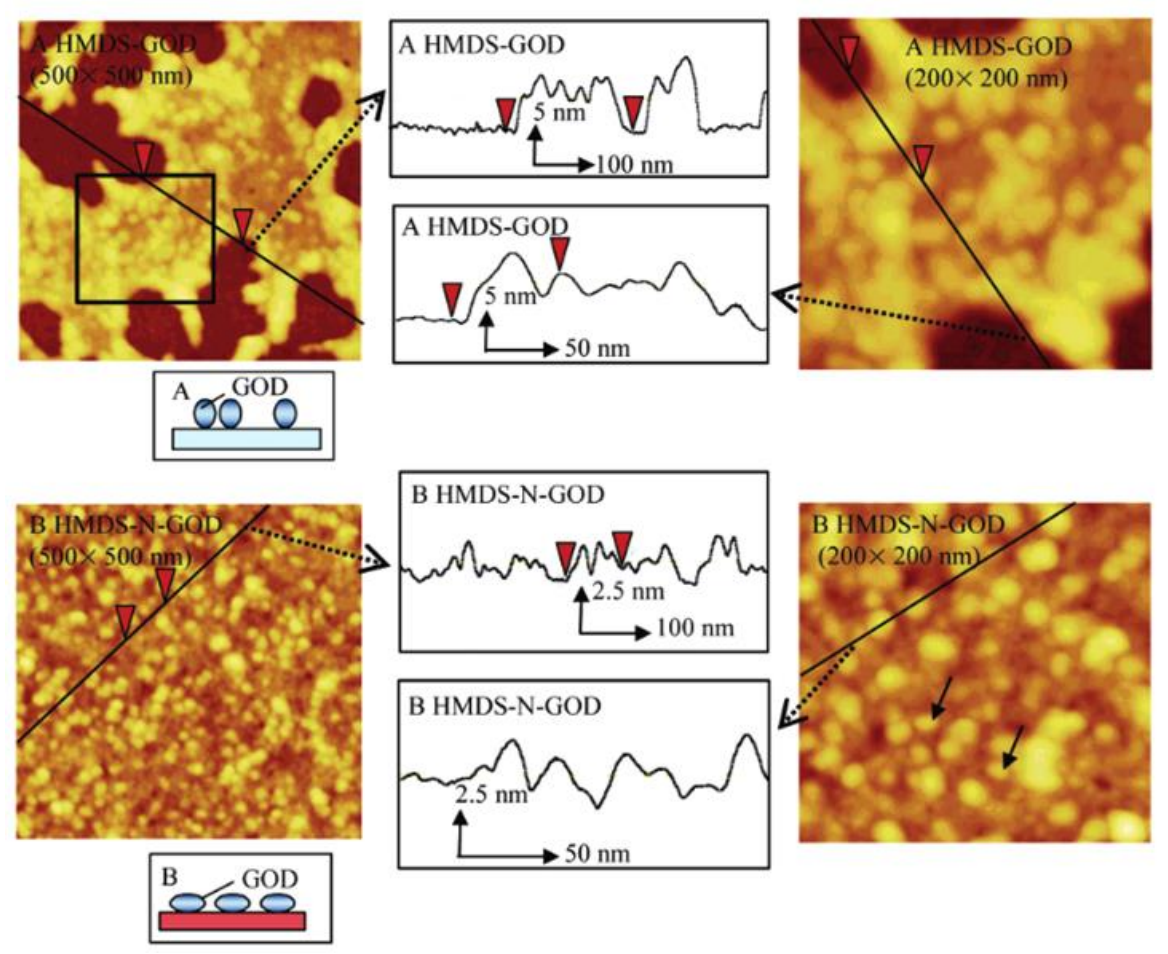

Figure 6- Tapping-mode AFM images and cross-sectional surface profiles of plasma-polymerized films (PPFs) of hexamethyldisiloxane (HMDS) after GOx adsorption. A- Hydrophobic and neutral pristine HMDS PPF (denoted HMDS-GOD on the figure); B- hydrophilic and positively charged HMDS PPF obtained by nitrogen plasma treatment (HMDS-N-GOD). The square in the 500-nm scale image of A (top left) corresponds to the enlarged 200-nm scale image view (top right). The arrows in B indicate a single GOx molecule. The red triangles in A and B correspond to those in the cross-sectional profiles. The lower insets in $A$ and $B$ are schematic illustrations of the enzyme orientation on the surface. Reproduced with permission from reference ${ }^{[89]}$

A homogeneous layer suggesting a uniform enzyme orientation due to specific binding was also observed by the same technique when glycosylated HRP was covalently bound on a mixed SAM. The covalent binding was realized via epoxy groups (boronate) while the affinity for sugar mannose likely ensured the specific orientation of the enzyme. ${ }^{[90]} A$ xanthine oxidase, with dimensions $17 \times 17 \times 9 \mathrm{~nm}^{3}$, was either adsorbed on gold or covalently linked on DTSP-modified gold via an outer lysine placed at the end of the longer axis. The resulting height distribution was studied by AFM and two results were observed. The height of $80 \%$ of the enzymes adsorbed on gold was between 5-9 nm, consistently with an orientation of the longer axis parallel to the surface. A wider distribution of heights was observed for immobilization on DTSP, with a maximum of enzymes showing a height between 14-15 nm, which indicated that the longer axis was perpendicular to the surface.$^{[91]} \mathrm{A}$ recombinant HRP was attached to a gold electrode via a tag situated at different positions with respect to the heme active site. Different height profiles of the proteins, observed by AFM measurement in liquid, indicated that different 
binding points lead to different orientations of the proteins. ${ }^{[92]}$ AFM also elucidated the orientation of the NiFeSe membrane-bound hydrogenase from Desulfovibrio vulgaris Hildenborough on a gold electrode modified with SAMs and a lipid bilayer. ${ }^{[93]}$ This hydrogenase is characterized by the presence of a lipidic tail at the opposite of the distal $4 \mathrm{Fe} 4 \mathrm{~S}$ cluster, the exit point of electrons. The enzyme is elongated and the longer dimension corresponds to the axis $4 \mathrm{Fe} 4 \mathrm{~S}$ cluster-lipidic tail. Depending on the immobilization method chosen, different orientations were observed. When the electrode, first modified with SAMs and a lipid bilayer, was incubated in a hydrogenase solution, the longer dimension was vertically oriented with the lipidic tail inserted in the lipid bilayer. On the contrary, when the electrode was co-incubated in a solution of hydrogenase and phospholipids, the 4Fe4S cluster was supposed to be oriented towards the electrode, while the hydrogenase lipidic tail was inserted in the lipidic bilayer on top of it. ${ }^{[93]}$ The same enzyme was combined with E. coli $\mathrm{F}_{1} \mathrm{~F}_{0}$ ATP-synthase inserted in the lipid bilayer overhanging it. The $F_{1} F_{0}$ ATP-synthase consists of a large soluble domain and a smaller membrane domain. AFM indicated a unique orientation of $F_{1} F_{0}$ ATP-synthase with its soluble domain directed towards the outer of the membrane. ${ }^{[94]}$ The bacterial respiratory complex I from Rhodothermus marinus, a L-shaped 2-domain protein, was reconstituted in a biomimetic membrane on a gold electrode. AFM evidenced that the hydrophilic part of the protein extended outside the lipidic membrane, and the recorded size suggested that the $L$ was flexible. ${ }^{[95]}$

\section{2- STM}

We could also find an example relying on the use of in situ STM. SM resolution of E. coli cyt.c NiR immobilized on $\mathrm{Au}(111)$ was achieved under electrochemical potential control in conditions of enzymatic electrocatalytic activity, and enabled among others the characterization of orientational distribution. A distribution of lengths characteristic of distribution of orientations was observed, which could also explain the very low $k_{\text {cat }}$ measured at the electrode, $30 \mathrm{~s}^{-1}$ compared to $770 \mathrm{~s}^{-1}$ in homogeneous medium. ${ }^{[73]}$

\section{3- SECM}

Finally, we report here two studies relying on SECM to characterize a preferential orientation enabling

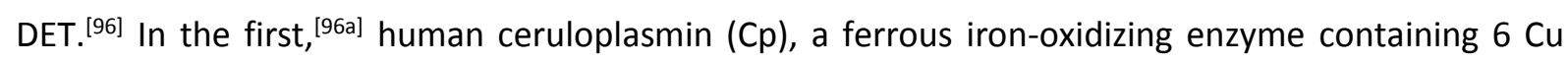
centers was immobilized at a gold electrode modified by magnetic nanoparticles. $\left[\mathrm{Fe}(\mathrm{CN})_{6}\right]^{4-}$ was oxidized by the enzyme immobilized on the sample electrode poised at $250 \mathrm{mV} \mathrm{vs.} \mathrm{Ag} / \mathrm{AgCl}$, a potential ensuring $\mathrm{Cu}$ oxidation in enzyme. The $\left[\mathrm{Fe}(\mathrm{CN})_{6}\right]^{3-}$ is detected at the tip UME poised at $80 \mathrm{mV}$ vs. $\mathrm{Ag} / \mathrm{AgCl}$. On bare gold, $\mathrm{Cp}$ is inactive towards ferrous iron oxidation; the highest activity is obtained in presence of nanoparticles and a magnetic field. The registered tip current is specific of $\mathrm{Cp}$ since it decreases in presence of $\mathrm{F}^{-}$, which is known to inhibit the enzyme. This result proves that the enzyme 
takes a specific orientation in the described conditions. Almost the same principle is applied in the second study, however the immobilization is realized in two steps. ${ }^{[96 b]}$ The orientation of cytochrome $c$ peroxidase $(\mathrm{CCP})$ is favored by controlling the orientation of its electron transfer partner cytochrome $c$ (cyt $c$ ) at an electrode and immobilizing the CcP by docking with cyt $c$. To immobilize this latter, two different covalent bindings are compared. The formation of amide bond between a SAM bearing carboxylic groups and lysine from the protein is tested but it rises two problems. It is not selective due to the presence of three lysines in the protein structure, and the binding could alter docking due the position of the most reactive lysine. On the contrary, the linkage between maleimide and a cysteine residue is selective since only one cysteine is present, and it should not alter the docking. The orientation of cyt $c$ is verified by SECM: a potential is applied to the electrode to reduce the cyt $c$, while the UME detects the oxidized $\left[\mathrm{Fe}(\mathrm{CN})_{6}\right]^{3-}$ formed by cyt $c$. The current at the UME is a measure of the DET between cyt $c$ and the electrode. The CCP is then immobilized by docking with cyt $c$. SECM in enzyme mediated feedback mode is further carried out to check the activity of immobilized enzyme. UME reduces benzoquinone (BQ) to hydroquinone ( $\mathrm{HQ}$ ), and $\mathrm{CCP}$ reduces $\mathrm{H}_{2} \mathrm{O}_{2}$ to $\mathrm{H}_{2} \mathrm{O}$ and oxidizes $\mathrm{HQ}$ to $\mathrm{BQ}$. A significant activity is recorded with the $2^{\text {nd }}$ strategy while it is very low in the absence of $\mathrm{CcP}$ or with the $1^{\text {st }}$ strategy.

\section{7- Imaging of local redox activity / Mapping heterogeneity}

Local redox enzyme activity was recorded in a live cell with conducting AFM ${ }^{[97]}$ and enzymatic activity in a single cell was also imaged using $\mathrm{SECM}^{[98]}$ We report here imaging of local activity of purified enzymes immobilized at the surface of an electron conductor in contact with an ionic conductor (electrolyte). We call therefore in the following this electron conductor an electrode although not all these surfaces are used as electrodes. The existence or not of an electron transfer, and its modality (direct or indirect electron transfer), influences on the choice of the method adopted to characterize the electrode. If direct electron transfer is established, activity mapping informs the experimenter about how the enzyme activity or its ability to exchange electrons are influenced by its localization, the inhomogeneity of the electrode surface or the inhomogeneity of enzyme immobilization. Purposes are somehow different if no electron transfer or only indirect electron transfer occurs. In these cases, the goal is rather to check if immobilization influences on enzyme catalytic activity, and if the substrate has access to the whole enzyme population. Many studies compare different immobilization methods.

The notion of heterogeneity in enzymatic catalysis has quite little been examined so far. In a few cases, the heterogeneity of enzymatic activity has been studied for enzymes immobilized at a surface via scanning probe microscopies. ${ }^{[25 d]}$ Mapping biocatalytically active surfaces was most of the times realized with SECM, in some cases in conjugation with a second technique. Contrary to AFM, SECM 
local study of enzyme mostly relies on the detection of enzyme activity. SECM correlates activity and localization, and permits to analyze enzyme activity at a well-defined location. Studies in SECM are not always conducted on conductive surface, due to technical problems for example with feedback-mode, but results could be easily transposed to the configuration where enzymes are connected in MET at electrodes. Of course, the issue is quite different when DET has to be established. SECM can be performed in different operating modes (described in part 1- Microscopy... and nanoscopy). We present first the case where no electron transfer occurs between the enzyme and the surface, with the following sub-division in three parts: 1- Feedback mode, 2- GC mode and 3- Hybrid methods. Then we present studies where electron transfer occurs, mostly realized in the redox competition mode. We describe the SM studies of enzymatic mechanism under electrochemical potential control in the next part (8- Mechanism, structure motion, conformation).

\section{1- No electron transfer between the enzyme and the underlying surface}

\subsection{1- SECM in Feedback mode}

Feedback mode was used very early to map enzyme activity. In a study by Heller, Bard and co-workers, the pores of a polycarbonate filter membrane were filled with a mixture of HRP and hydrogel. The activity was recorded by SECM in enzyme-mediated feedback mode. Hydroquinone electro-generated at the tip from benzoquinone in solution was catalytically re-oxidized to benzoquinone by HRP in the presence of $\mathrm{H}_{2} \mathrm{O}_{2}$. High current regions corresponding to pore dimensions were observed and surrounded by rings with lower currents. An avidin-labeled hydrogel was also immobilized on a glass slide, and biotin-labeled HRP was conjugated to the avidin in the film. The SECM feedback current was a linear function of the HRP surface concentration. ${ }^{[99]}$ Biotinylated PQQ-dependent glucose dehydrogenase $(\mathrm{GDH})$ bound to streptavidin-coated paramagnetic beads were deposited as microspots at a surface. After finding the location of micro spots of active enzyme on the surface, the authors elucidated kinetics of the immobilized enzymes. The procedure enables quantitative analysis of GDH catalysis. First the analysis was conducted in enzyme mediated feedback mode, which necessitates high surface enzyme concentrations. The difference of size of spots in optical microscopy (around $100 \mu \mathrm{m}$ ) and in SECM (around $150 \mu \mathrm{m}$ ) was attributed to some blurring due to products diffusion. Sensitivity was enhanced in GC mode compared to feedback mode, however the lateral resolution was lower and the spots appeared even bigger. Another interesting input of the study is the demonstration that the mediator choice influences the sensitivity. ${ }^{[100]}$ Planar electrodes and more complex matrices can be imaged, and SECM can be coupled to other methods to deconvolute topographical and reactivity information. To eliminate topographic artifacts, AFM and SECM observations were coupled. Lactate oxidase simply adsorbed on HOPG or glassy carbon was 
characterized by AFM under liquid conditions, while spatially resolved mapping of enzymatic activity was obtained by SECM. SECM was used in feedback mode, following the oxidation of the mediator at the tip in the presence or absence of lactate, to determine the influence of immobilization on morphological and catalytic properties of the enzyme. The combination of AFM, SECM and cyclic voltammetry was used to correlate deposition procedures to changes in morphology and biological activity. Spatially inhomogeneous enzyme activity was observed. ${ }^{[20]} \mathrm{A}$ sol-gel film containing BOD and laccase was also imaged by several techniques: SECM and CLSM. The enzyme distribution and activity were studied by SECM in feedback mode with ferricyanure as a mediator and a Au UME. Approach curves were recorded at the center and at the edges of the film, showing that laccase displays a higher activity and higher concentrations at the borders of the film. Moreover, CLSM indicated that the film thickness was increased at the edges due to edge effect. ${ }^{[101]}$ In another study, hybrid scanning ion conductance microscopy (SICM)-SECM enabled resolving the distribution of activity of enzyme spots containing HRP and GOx on an uneven surface with sub-micrometric resolution. The probe was a nanoring electrode surrounding a nanopipette for simultaneous recording of topography and electrochemical reactivity. SECM was conducted in SG/TC mode for HRP and in feedback mode for GOx. Topographic and activity images were in good agreement, displaying a ringlike structure with higher activity at the edges. ${ }^{[102]}$

SECM mainly uses UME so typically a micrometric resolution is reached. In the last few years, nanoscale SECM, which requires fabrication of geometrically well-defined nanoelectrodes from different materials (mainly platinum, although carbon's importance cannot be denied ${ }^{[27 b, 103]}$ ), has been developed. ${ }^{[27 b]}$ The nanoscale tips show enhanced transport rates, lower double-layer capacitances, and better $\mathrm{S} / \mathrm{N}$ ratios. Pt nanoelectrodes with a diameter around $120 \mathrm{~nm}$ have been developed to map activity of immobilized enzymes at the nanoscale level. Lactate oxidase (LOx) adsorbed on bare gold or covalently attached to SAM on gold has been investigated in enzyme-mediated feedback mode in the presence or absence of lactate. In the case of enzyme adsorption, clear peaks were observed, indicating the relative positions of catalytically active LOx molecules. The small amount of functional enzyme was attributed to activity loss upon adsorption. In the case of covalent binding, enzymes were much more densely packed and their activity was observed over columns or arrows. The higher total activity indicated less denaturation. ${ }^{[104]}$

\subsection{2- SECM in GC mode}

SECM in GC mode has for example enabled imaging micrometer-sized spots of GOx covalently anchored on Au. Defects were introduced in a SAM, and new functionalities were introduced in these defects to allow $\mathrm{GOx}$ covalent anchorage. $\mathrm{H}_{2} \mathrm{O}_{2}$ generated by the enzymatic reaction at these sites was 
collected by the platinum tip in the SG/TC mode. ${ }^{[105]}$ In a further study, local imaging of enzymatic activity of HRP micropatterns periodically disposed was realized, and immobilization procedures to better retain the enzyme activity were evaluated. Micropatterns of enzyme mixtures containing HRP or GOx were obtained on chemically modified surfaces (glass or gold) by different soft-lithographic strategies. SECM evidenced patterns of $100 \times 100 \mu \mathrm{m}^{2}$ separated by $50 \mu \mathrm{m}$ intervals (Figure 7). ${ }^{[106]}$ Similarly, SECM was used in TG/SC mode to screen oxygen reduction activity on arrays of "wired" enzyme spots, BOD and laccase, on a carbon electrode for optimization of the polymer/enzyme mixture. ${ }^{[107]}$ Enzymes were immobilized on the electrode surface in an Osmium-polymer matrix whose ratio varied within the array. Using the GC mode, a gold UME tip biased positively to produce $\mathrm{O}_{2}$ from water was scanned above the enzymatic array. The authors found the optimal weight ratio for the two enzymes at which the oxygen reduction rate was close to the diffusion limit. SECM saves time and material compared to conventional methods like the rotating disk electrode to analyze different systems. ${ }^{[107]}$ SECM imaging of photosystem I (PSI) adsorbed on SAM film on a micro-patterned gold surface proves selective adsorption of PSI onto hydroxyl-terminated SAM rather than the methylterminated SAM. ${ }^{[108]}$ On planar surfaces, SECM in GC mode was used to characterize local activity and stability of HRP and GOx covalently bound on transparent and biocompatible $\mathrm{TiO}_{2}$ semi-conductor surface chemically or electrochemically generated. The method allowed comparing the efficiency of enzyme attachment on the $\mathrm{TiO}_{2}$ prepared in two different manners because currents recorded at the UME significantly differed between the two surfaces. ${ }^{[60]}$ In another case, reduction of ferrocinium methanol produced during enzymatic $\mathrm{H}_{2} \mathrm{O}_{2}$ reduction was measured at a $300 \mathrm{~nm}$ radius $\mathrm{Pt}$ probe to image the distribution of enzymatic activity of HRP covalently attached over individual Au nanowires (2-3 $\mu \mathrm{m}$ long and $140 \mathrm{~nm}$ in diameter). Enzyme activity was detected all over the individual wires but was more important at the ends. This was consistent with AFM observations that evidenced larger concentrations of HRP molecules at the nanowires extremities (Figure 7). ${ }^{[109]}$ 

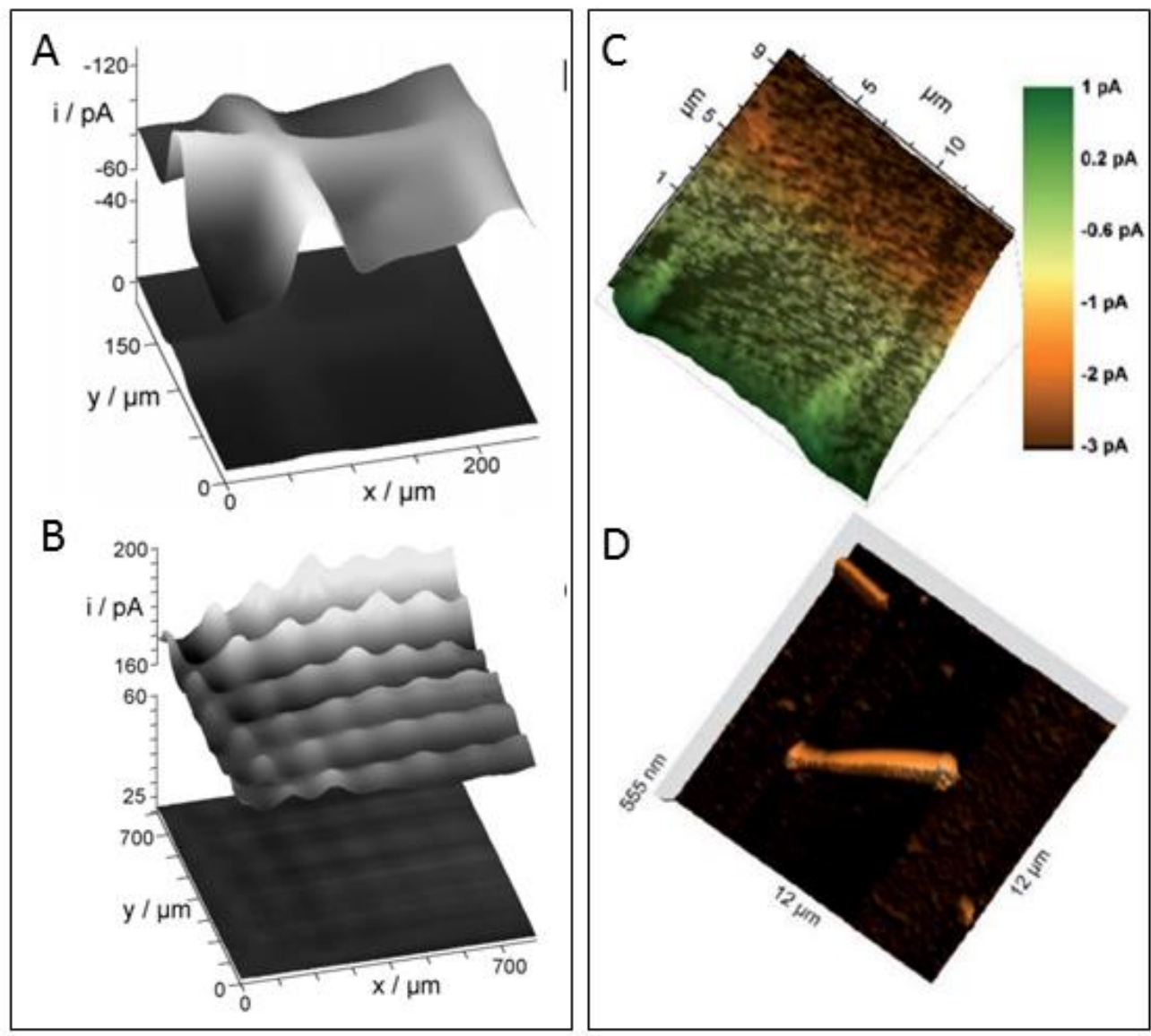

Figure 7- SECM imaging of localized enzymatic activity. Left panel: Localized HRP (A) and GOX (B) activity on patterned monolayers on gold (A) or glass (B); Right panel: Images of individual HRP-linked AuNWs immobilized on a gold-coated glass slide. In A and B, patterns are squares of $100 \times 100 \mu \mathrm{m}^{2}$ separated by $50 \mu \mathrm{m}$-wide lines. HRP was bound to the lines between the squares (A), and GOX was bound to the squares (B). (A) HRP activity recorded in $\mathrm{GC}$ mode in a solution of $5 \mathrm{mM}$ hydroxymethylferrocene $+0.1 \mathrm{M} \mathrm{KCl}$ with (top surface) or without (bottom surface) $0.5 \mathrm{mM} \mathrm{H}_{2} \mathrm{O}_{2} . r_{\mathrm{TIP}}$ $=12.5 \mu \mathrm{m}, E_{\mathrm{TIP}}=0 \mathrm{mV}$ (B) GOX activity recorded in $\mathrm{GC}$ mode in air-saturated phosphate buffer, $\mathrm{pH} 7$ with (top surface) or without (bottom surface) $50 \mathrm{mM}$ glucose. $r_{\mathrm{TIP}}=12.5 \mu \mathrm{m}, E_{\mathrm{TIP}}=750 \mathrm{mV}$. (C) $\mathrm{SECM}$ in SG/TC mode at $E_{\text {probe }}=0 \mathrm{~V}$ probe potential after the addition of the enzymatic substrate $\mathrm{H}_{2} \mathrm{O}_{2}$. The images were recorded with a $300 \mathrm{~nm}$ radius probe at a scan rate of $5 \mathrm{~mm} \mathrm{~s}^{-1}$ in $1.5 \mathrm{mM}$ ferrocenemethanol solution in $\mathrm{pH}$ 6. (D) Representative AFM image of the same substrate as in C. Reproduced with permission from reference ${ }^{[106]}$ and ${ }^{[109]}$

Specifically modifying the probes can enhance their selectivity. Modifying a UME tip with Prussian blue to characterize the activity of GOx adsorbed on platinum by SECM in GC mode enhanced sensitivity, spatial resolution and image quality. ${ }^{[110]}$ The same electrode modification was conducted to map biocatalytical activity of lactate oxidase immobilized in various polymer matrices. Micrometric spots of lactate oxidase-containing siloxane sol-gel were deposited on carbon paste materials. The influence of 
sol-gel density on the activity of the contained enzyme was studied to search for optimal immobilization conditions. The carbon paste UME SECM tip was modified with Prussian blue for more sensitive detection of $\mathrm{H}_{2} \mathrm{O}_{2}$ formed during the enzymatic reaction in the presence of lactate. To avoid topographic artifacts, imaging was moreover performed in contact mode with a soft probe. Soft microelectrodes indeed enable contact scanning at constant distance, without risk of damaging the sample or the tip. ${ }^{[111]}$ More recently, the detection of local hydrogen evolution by a hybrid biocatalyst based on PSI and platinum nanoparticles immobilized in a redox polymer on a gold electrode has been performed by scanning photo-electrochemical microscopy using a hydrogenase-modified microelectrode. Briefly, a mixture of hydrogenase and viologen-modified redox polymer was immobilized at a carbon microelectrode inserted in a glass capillary, which upon scanning ensured both the local illumination of the sample and the local detection of $\mathrm{H}_{2}$ (Figure 8). ${ }^{[112]}$
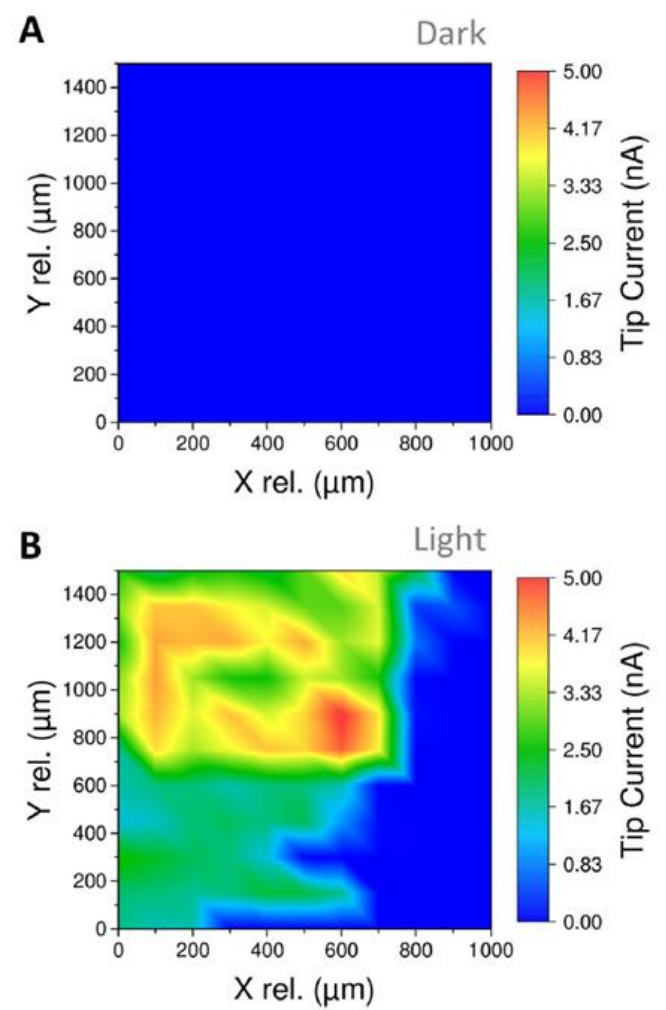

Figure 8- Scanning photo-electrochemical microscopy (SPECM) scans of a spot of PSI and platinum nanoparticles immobilized in a osmium-complex redox hydrogel partially covering the gold sample surface. The frames show the hydrogen oxidation current recorded at the SPECM tip modified by the mixture of Desulfovibrio vulgaris Miyazaki F [NiFe]-Hydrogenase and viologen redox polymer A- in the dark and B- under localized illumination of the sample through the positioned SPECM tip. Electrolyte is Ar-saturated pH 5.0 phosphate-citrate buffer. Both tip and gold sample surface are polarized at $0 \mathrm{~V}$ vs. $\mathrm{Ag} / \mathrm{AgCl} / 3 \mathrm{M} \mathrm{KCl}$. Reproduced with permission from reference ${ }^{[112]}$ 
We wish to introduce here a last example relying on the same technique and proving the interest of microscopy, although it does not fit in any part of the adopted plan: the characterization of diffusion layer of substrates and products of enzymatic reaction (Figure 9). ${ }^{[113]}$ This was realized for a glucose oxidase (GOx), which couples the oxidation of glucose into gluconolactone with the reduction of $\mathrm{O}_{2}$ into $\mathrm{H}_{2} \mathrm{O}_{2}$. After $\mathrm{GOx}$ immobilization on an insulating surface, $\mathrm{SECM}$ in $\mathrm{GC}$ mode or Redox-competition mode allowed characterizing respectively where $\mathrm{H}_{2} \mathrm{O}_{2}$ diffuses and where $\mathrm{O}_{2}$ is consumed. Variations on the range $[0-100 \mu \mathrm{m}]$ from the surface were found in $\mathrm{RC}$ mode for local $\mathrm{O}_{2}$ consumption by $\mathrm{GOx}$ in the presence of glucose. Beyond $100 \mu \mathrm{m}$, differences in $\mathrm{O}_{2}$ concentrations compared to what is observed in the absence of glucose are very slight. $\mathrm{H}_{2} \mathrm{O}_{2}$ diffusion extends much further since at 10 mmol. $\mathrm{L}^{-1}$ glucose, $\mathrm{H}_{2} \mathrm{O}_{2}$ production is seen in $\mathrm{GC}$ mode until $600 \mu \mathrm{m}$ from the surface. Although not realized under electrochemical potential control, this study gives clues about the extent of the diffusion layer in catalysis by immobilized enzymes, which is also of major importance for electrochemical phenomena.
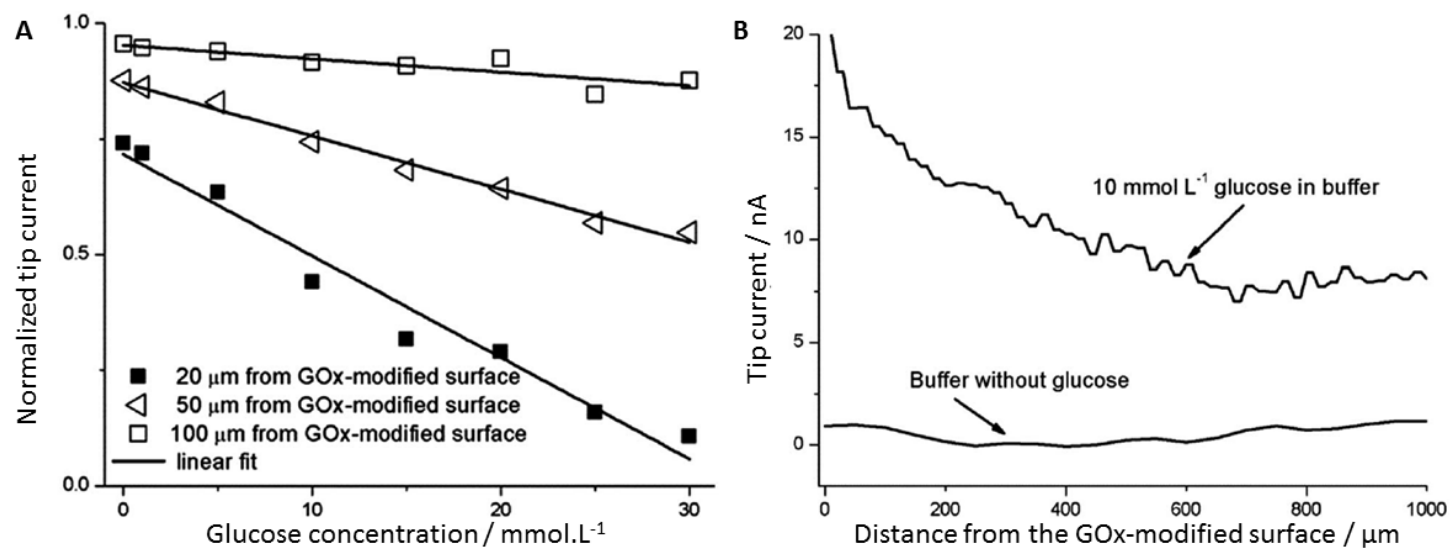

Figure 9-Spatial distributions of a reactant $\left(\mathrm{O}_{2}\right)$ and a product $\left(\mathrm{H}_{2} \mathrm{O}_{2}\right)$ during glucose oxidation catalyzed by an immobilized GOx. A- Dependence of $\mathrm{O}_{2}$ reduction current at the UME tip held at $\mathrm{E}_{\text {tip }}=-0.6 \mathrm{~V}$ vs. $\mathrm{Ag} / \mathrm{AgCl}$ on glucose concentration at different tip distances from the GOx-modified surface. $\mathrm{B}-\mathrm{H}_{2} \mathrm{O}_{2}$ concentration profile obtained by recording $\mathrm{H}_{2} \mathrm{O}_{2}$ oxidation current at the UME tip held at $\mathrm{E}_{\text {tip }}=+0.6 \mathrm{~V}$ vs. $\mathrm{Ag} / \mathrm{AgCl}$ when approaching the GOx-modified surface in the presence or absence of glucose. Reproduced with permission from reference ${ }^{[113]}$

\subsection{3- Hybrid methods}

Alternatively, hybrid probes can be designed to enable simultaneous recording of electrochemical and atomic-force signals. The drawback of SECM compared to other SPM is the lack of sufficient lateral resolution due to signal-dependent positioning of the micro-electrode and the convolution of electrochemical response to topological information by scanning at constant height. An AFM probe with integrated nano- and sub-micro electrodes enables simultaneous AFM and SCEM imaging 
(combined AFM-SECM) and deconvolution of the two signals. Detection of the electroactive byproduct at the micro-electrode is a signature of enzymatic activity while AFM in contact mode enables topography determination. ${ }^{[114]}$ Micro-patterns $(1-2 \mu \mathrm{m})$ of isolated enzyme spots were formed by covalent binding on periodically patterned silicon nitride layer deposited onto a gold-coated silicon wafer. The corresponding HRP activity was localized at the electrode during turnover by topographical and activity imaging in a single time and space correlated measurement. The re-shaped AFM tip was located in the center of the frame electrode. AFM was conducted in contact mode while SECM simultaneously enabled detection of ferrocinium methylhydroxide $\left(\mathrm{FCMH}^{+}\right)$, the electroactive byproduct of the enzymatic reaction. ${ }^{[115]}$ Another method, inspired by a work by Bard and coworkers, ${ }^{[116]}$ consisted in coupling SECM with continuous nano-flow ${ }^{[117]}$ to overcome a general problem linked to the SG/TC mode: the increasing background current resulting from steady build-up of the reaction products in the diffusion layer. At long experience times, activity spots are not anymore distinguishable from the background. This study did not deal strictly speaking with an enzymatic electrode since it consisted in mapping the repartition of immobilized IgG. However the detection relied on enzymatic activity: IgG spots were covered with HRP-labelled anti IgG. The authors designed a beveled micro-concentric Au ring electrode (10.9-11.5 $\mu \mathrm{m})$ which contains an inner injector probe (diameter $5.5 \mu \mathrm{m}$ ) to deliver substrate $\mathrm{H}_{2} \mathrm{O}_{2}$ and mediator FCMH in the center of the SECM probe. The solution flows radially out of the probe so that only the spot imaged is submitted to sufficient concentrations and thus activated. This lead to higher imaging resolution because no interference from other active sites, which causes overlapping of the diffusion layer, was observed, neither interference from the bulk (Figure 10). Moreover, the possibility to put whatever is needed in the capillary ensures the versatility of the method. ${ }^{[117]}$ 


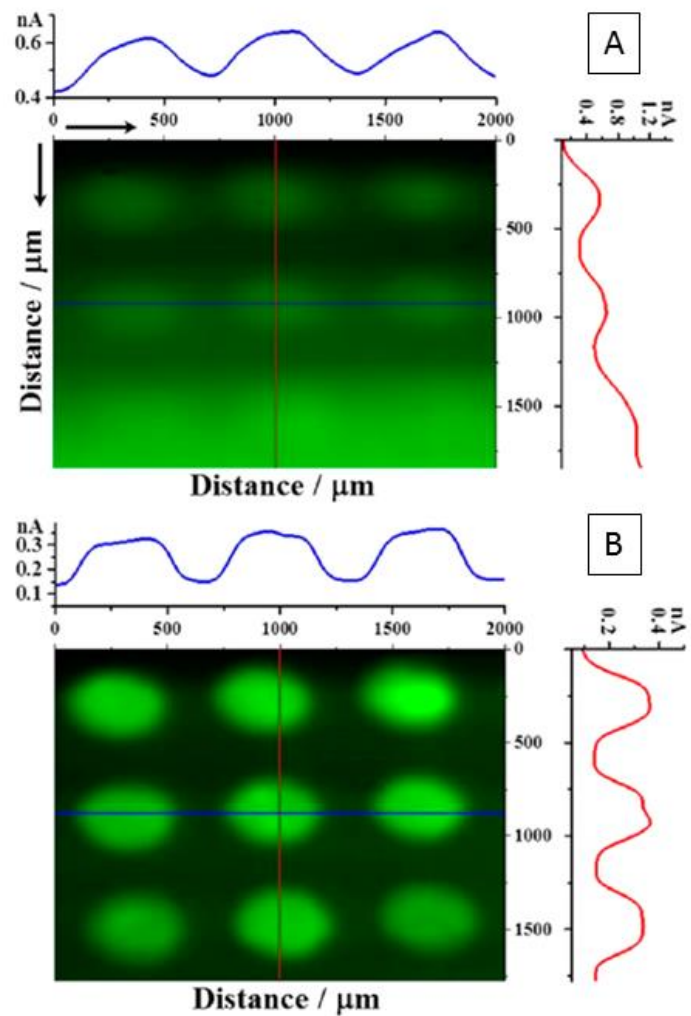

Figure 10- Comparison between conventional SECM in GC mode and continuous nano-flow SECM (CNFSECM). A- SECM image of a microarray of HRP-labeled spots. The image is collected by measuring at the SECM probe the reduction current at $0.0 \mathrm{~V}$ of ferrocinium-methanol formed during the enzymatic

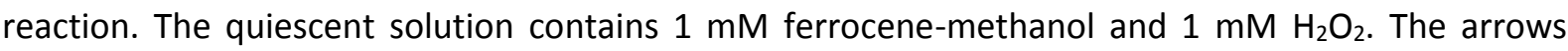
indicate the SECM rastering directions. B- Same region mapped by CNF-SECM. The measurement of ferrocinium-methanol reduction is coupled to continuous flow of $1 \mathrm{mM}$ Ferrocene-ethanol and $1 \mathrm{mM}$ $\mathrm{H}_{2} \mathrm{O}_{2}$ at $15 \mathrm{~nL} / \mathrm{min}$ into $250 \mu \mathrm{L}$ of phosphate buffer through the CNF-SECM probe. The top and right cross-sectional contours correspond to the spots intersecting with the blue and red lines, respectively. Reproduced with permission from reference ${ }^{[117]}$

In tip generation/substrate collection mode, SECM enables locally triggering enzymatic activity. This is also possible thanks to combination of a scanning microelectrode and light emitting methods, like fluorescence or ECL. Local activity controlled via a microelectrode can be recorded via fluorescence. In a case where alcohol dehydrogenase $(A D H)$ immobilized on agarose beads on glass presented a maximal activity at $\mathrm{pH} 9$, the microelectrode locally reduced $\mathrm{O}_{2}$, which locally increased the $\mathrm{pH}$ initially set to 6 and thus the enzyme activity. The enzymatic reaction oxidizes ethanol to ethanal and reduces $\mathrm{NAD}^{+}$to $\mathrm{NADH}$ which is excited maximally at 340-360 nm and emits at $460 \mathrm{~nm}$. The local activity enhancement was recorded by following the fluorescence of the NADH cofactor. To increase the spatial resolution, the authors added pyrophosphate buffer to the electrolyte to restrict the expansion of the zone of $\mathrm{pH}$ change. The highest resolution achieved was 7-12 $\mu \mathrm{m} \cdot{ }^{[118]}$ SECM and scanning 
electrochemiluminescence ( $E C L$ ) have been combined to visualize the locally triggered enzymatic activity of spots made of glucose oxidase and polymer on transparent surfaces. The luminol chromophore was locally oxidized thanks to the SECM tip and a photomultiplier tube (PMT) was placed underneath the 3-electrode setup to record the ECL emission. GOx produced locally $\mathrm{H}_{2} \mathrm{O}_{2}$ which was used in the ECL reaction with luminol. Measurements of local enzyme activity were similar to those obtained in SECM, with variations that were attributed to the different mechanisms involved in signal generation. Inhomogeneous activity was observed in the enzyme-polymer spot, with a higher activity in the ring and a smaller one in the center (Figure 11). ${ }^{[119]}$

A
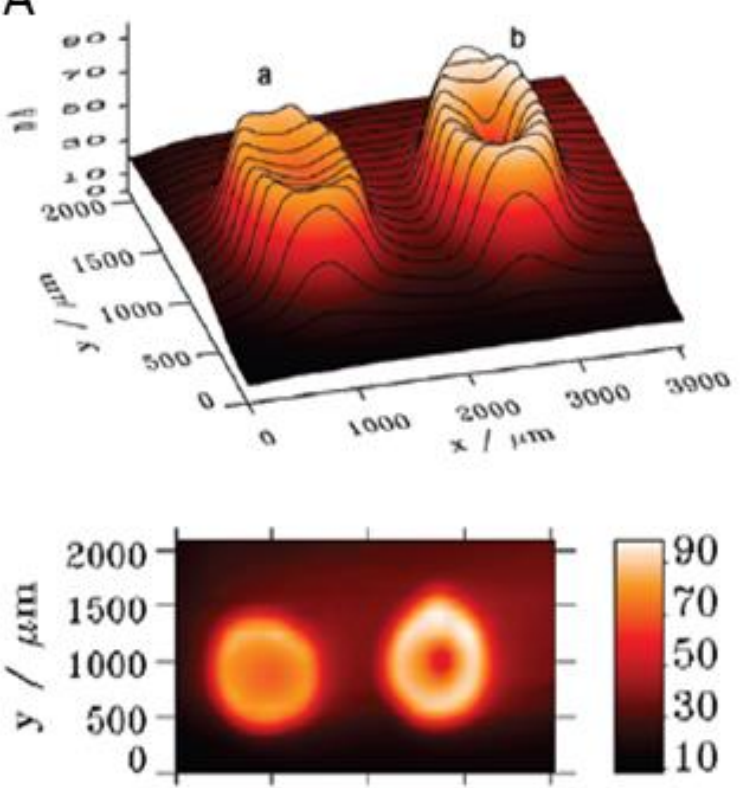

B
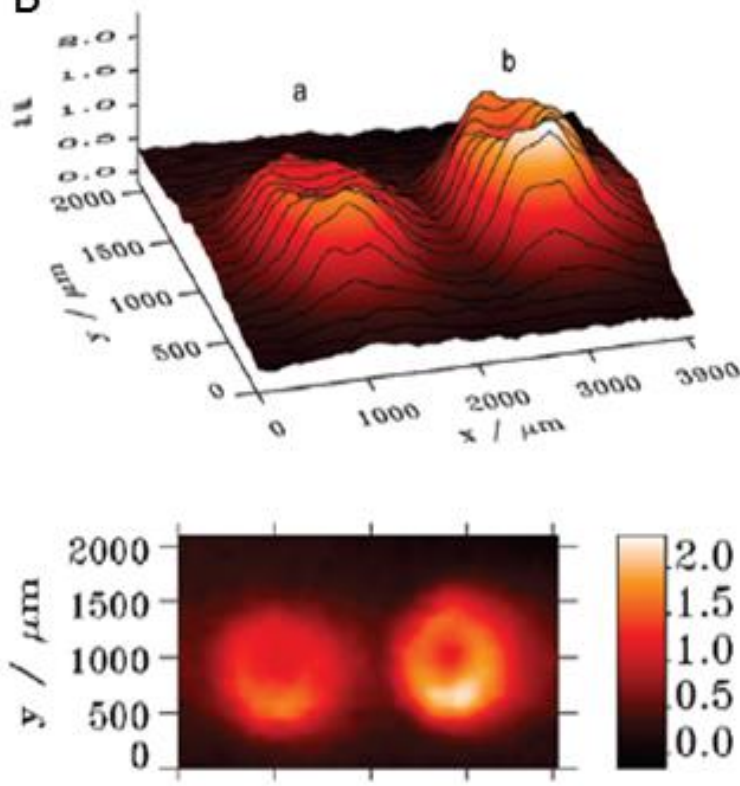

Figure 11: Images of the enzymatic activity of spots made of a mixture of GOx and polymer by means of SECM in GC mode (A) and scanning electrochemiluminescence (SECL) (B). (A) GC-SECM image of GOx-polymer spots in $100 \mathrm{mM}$ glucose and $0.20 \mathrm{M}$ phosphate buffer (pH 6.8); (B) SECL image of the same spots as SECM in $100 \mathrm{mM}$ glucose, $1 \mathrm{mM}$ luminol, and $0.20 \mathrm{M}$ Tris- $\mathrm{HCl}$ buffer (pH 8.5) using a 250 $\mu \mathrm{m}$ Pt disk as tip electrode. Reproduced with permission from reference ${ }^{[119]}$

\section{2- Electron transfer between the enzyme and the underlying surface}

Before concluding this part, we wish to mention the rare scanning probe mappings of enzymatic activity performed under electrochemical control, i.e. while the immobilized enzyme was submitted to the electrode potential and could exchange electrons with the electrode. In SECM, either TG/SC or the redox competition mode proposed by Eckhard and Schuhmann ${ }^{[31]}$ can be used. In both modes, a signal is recorded only if the enzyme is active and can exchange electrons with the electrode on which it is immobilized. In feedback mode, an electron transfer to this electrode would interfere with imaging of enzymatic activity. SECM in redox competition mode was first proposed in a biocatalytic context for a 
BOD embedded in an Osmium polymer on a carbon chip. ${ }^{[31 b]}$ In this study, the enzyme was entrapped in a polymer obtained by electrochemically-induced deposition. During catalysis, electrons were transferred indirectly between the enzyme and the electrode via hopping between the neighboring redox active osmium sites. By poising both the tip and the carbon chip at a convenient potential, $\mathrm{O}_{2}$ reduction at the tip is in competition with $\mathrm{O}_{2}$ reduction by the BOD, which allows to record spatiallyresolved BOD activity. In the case of laccase embedded in sol-gel films with ${ }^{[120]}$ or without ${ }^{[121]}$ carbon nanoparticles, direct electron transfer with the electrode was established. Water was first oxidized at the Pt UME, leading to $\mathrm{O}_{2}$ generation. The probe platinum UME and the enzyme film were then competing for $\mathrm{O}_{2}$ reduction. In the absence of CNPs laccase aggregation was observed, as well as inhomogeneity. ${ }^{[121]}$ In their presence, control experiments were conducted to take into account topographical features that could affect $\mathrm{O}_{2}$ diffusion. A homogeneous activity was recorded, demonstrating a homogeneous enzyme distribution. ${ }^{[120]}$

\section{8- Mechanism, structure motion, conformation}

Finally, single molecule methods enabled to image single enzymes during catalytic operation. Differences were observed between inactive state and turnover, ${ }^{[73,122]}$ or even different steps of a catalytic cycle could be resolved. ${ }^{[123]}$ STM and AFM imaging of biomolecules were realized at electrochemical interface in the context of protein monolayer voltammetry (PMV) on modified gold, to understand molecular electron transfer and proton transfer process. Most of the time single crystal electrodes were used to enhance the S/N ratio in STM. ${ }^{[124]}$ The interest to consider single enzyme molecules is to provide a distribution of conformational heterogeneities, and reaction mechanism that are not observed otherwise due to averaging. However, SM or SP imaging remains a challenge. ${ }^{[125]}$ In most studies described here, enzyme was in direct electronic contact with the electrode.

\section{1- STM}

Scanning tunneling microscopy (STM) was developed in situ during electrochemical enzymatic turnover, ${ }^{[73,122]}$ enabling single enzyme molecule observation and especially observation of intramolecular ET triggered by potential or substrate binding. ${ }^{[122]}$ In electrochemically-controlled STM (EC-STM), both sample electrode and tip are under electrochemical potential control relative to a common reference in aqueous buffer. This is particularly suitable for in situ mapping of electronic structures and electronic properties of redox proteins during their biological action such as electron transfer or electrocatalysis. Particularly interesting are multi-center redox enzymes like the trimeric copper nitrite reductase from Achromobacter xylosoxidans (Ax NiR), a homotrimer containing $2 \mathrm{Cu}$ centers per molecule. The T1 Cu acts as an electron relay, while the T2 Cu center is the place of catalytic $\mathrm{NO}_{2}^{-}$reduction. Ax NiR was immobilized on Au (111) and studied by in situ STM at the SM level during 
catalytic action. Molecular scale contrast was observed only in presence of nitrite. ${ }^{[126]}$ In a further study, the monocopper-containing azurin and Ax NiR were "wired" at the electrode using a piconjugated thiol for observation of long-range interfacial electron transfer at the nanoscale and singlemolecule level, as well as intramolecular electron transfer in the case of $A x$ NiR. NiR individual molecules could be observed although with a smaller resolution than for azurin, because the size of the enzyme implies a large tunneling barrier. The SM contrast of azurin was clearly tuned by redox state of the protein, while nitrite was essential to obtain detectable Ax NiR SM contrast. This suggests that nitrite serves as a gate for long-range interfacial electron transfer, introducing the notion of "substrate gated electron transfer". This added to observations in PMV suggest that binding of nitrite to the enzyme is necessary to ET by opening the tunneling pathway. Intramolecular electron transfer presupposes "cooperativity" between the redox centers. Here the rate of intramolecular ET is modified by substrate binding, or substrate binding triggers changes in the T2 Cu redox potential thus modifying the driving force of the electron transfer reaction. Substrate binding could also enhance the conductivity of enzyme molecules. ${ }^{[72]}$ STM description at the molecular level under electrochemical potential control in conditions of enzymatic electrocatalytic activity was also achieved for the cytochrome $c$ nitrite reductase from $E$. coli ( $E c$ cyt.c NiR), a decaheme-containing homodimer that catalyzes the 6-e reduction of nitrite into ammonium. Contributions from enzyme molecules with nonuniform properties could be recorded in constant current mode to establish a map of electrontunneling properties of the protein/electrode interface. Vertical projection of an imaged molecule represents tunneling current determined by electronic properties rather than topographical features. ${ }^{[73]}$ Similarly, laccase immobilized on a SAM-modified Au electrode with direct electronic contact could be observed at the SM resolution during electrocatalytic action. An intramolecular electron transfer triggered by potential or by $\mathrm{O}_{2}$ binding was observed: reducing potentials open an efficient electron-tunneling channel through the enzyme. This potential-induced electron structural change in the enzyme is reversible. Moreover, the presence of pure $\mathrm{O}_{2}$ rather than air induces a stronger contrast (Figure 12). These observations give clues concerning the mechanism of the immobilized enzyme and its interfacial behavior. ${ }^{[122]}$ 

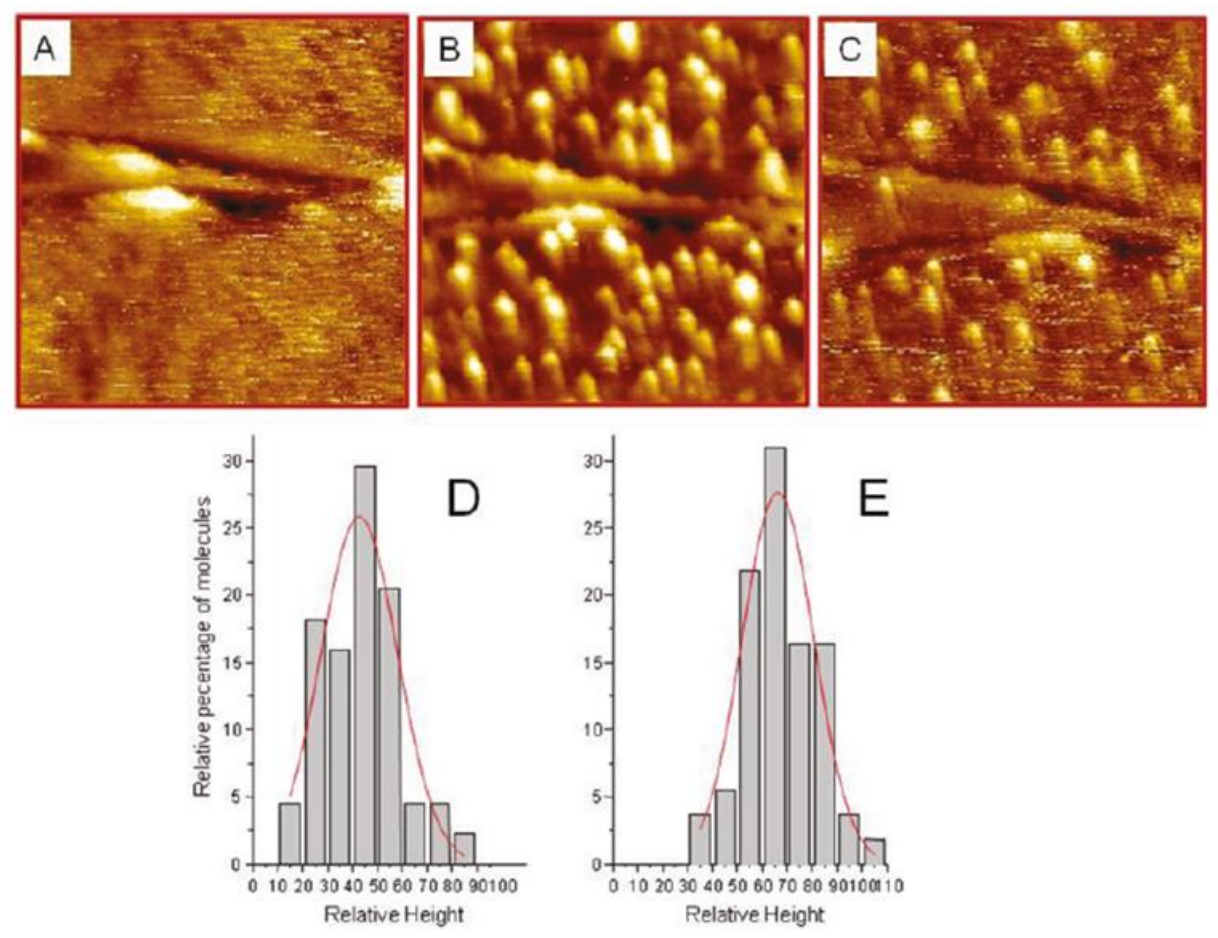

Figure 12- In situ STM images of laccase from Streptomyces coelicolor immobilized on gold (111) modified with butane-thiol under pure oxygen atmosphere. A- Initial image at a potential at which no catalytic current is observed ( $0.84 \mathrm{~V}$ vs the reversible hydrogen reference electrode (RHE)): enzyme cannot be distinguished. Bias voltage: $-0.70 \mathrm{~V}$. B- Image after decreasing the potential in the catalytic potential region (0.44 V vs RHE): enzyme appear as bright spots. Bias voltage: $-0.30 \mathrm{~V}$. C- Image after returning at $0.84 \mathrm{~V}$ vs RHE: the bright spots do not completely disappear. Bias: $-0.70 \mathrm{~V}$. The tip potential was kept constant so that the bias voltage was varying. The scan area was $100 \times 100 \mathrm{~nm}^{2}$. Histograms $D-$ and $E-$ show the height distribution of the bright spots in (B) and (C), respectively. Height was normalized against the maximum height measured in (B). Lines are best fit to a normal distribution. Reproduced with permission from reference ${ }^{[122]}$

\section{2- $A F M$}

Electrochemically controlled in situ AFM was also developed to address specifically single-molecule metalloenzymes and get a direct view on structural changes of the working enzyme under potential control in presence of substrate. ${ }^{[123 a]} A x$ Nitrite reductase was immobilized on gold modified by cysteamine and direct electronic connection was established. The evolution of height of single molecules during cyclic voltammetry was followed by AFM in situ with a nanometric tip ( $2 \mathrm{~nm}$ ). A dense but disordered monolayer of molecular-scale structures stable over the potential range was observed. The size of the molecules was found to change upon potential scanning, from $4.5 \mathrm{~nm}$ in the oxidized ${ }^{[124]}$ state to $5.5 \mathrm{~nm}$ in the reduced state, exclusively in the presence of nitrite substrate. This observation suggests conformational change upon substrate binding, and significant conformational change when 
electrocatalysis sets in upon potential scanning. ${ }^{[123 a]}$ We would like to mention also an interesting contribution concerning cellobiose dehydrogenase immobilized on gold, although in this case no electron exchange occurred between enzyme and gold. ${ }^{[123 b]}$ The enzyme consists of two domains connected via a flexible linker. The "cytochrome domain" contains a heme: the apo-enzyme was immobilized and specifically oriented on gold by reconstituting the enzyme on a heme covalently linked to the surface. The protein reconstitution was visualized by high speed AFM (HS-AFM). The other domain, the "dehydrogenase domain" contains a FAD. HS-AFM, with sub-second time scale and submolecular lateral resolution, enabled to visualize the interdomain flip-flop motion. Upon addition of cellobiose, it becomes clear that one domain is fixed at the surface (the cytochrome domain, since it is anchored) while the other (the FAD domain) moves rapidly around it (Figure 12). The motion intensity increases with increasing cellobiose concentrations.

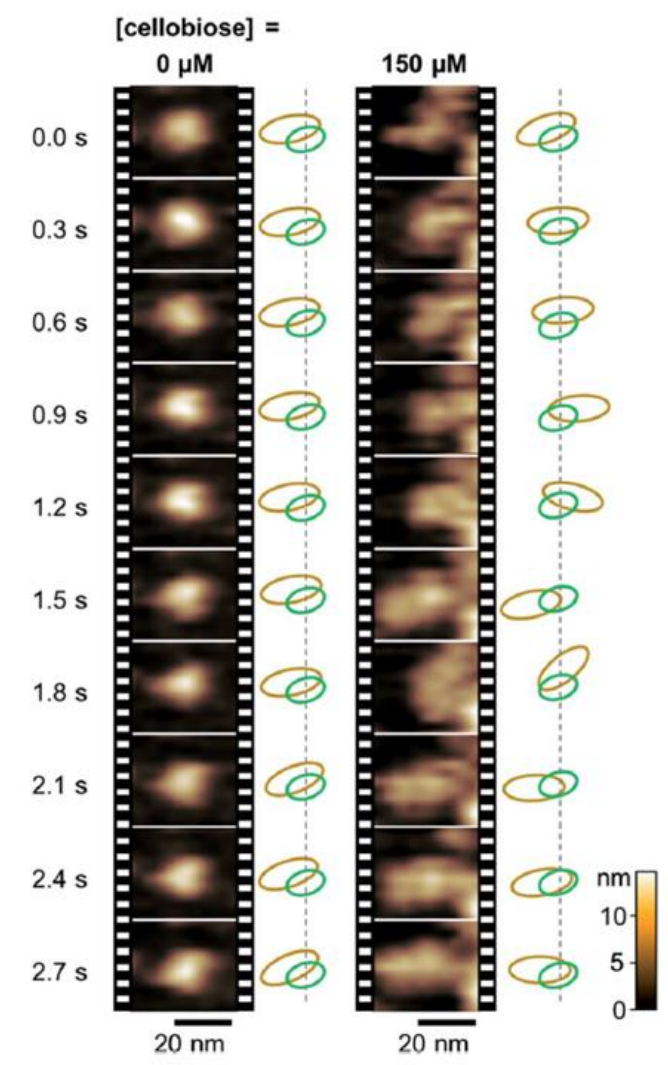

Figure 12- Real-time observation by high-speed AFM of Phanerochaete chrysosporium flavocytochrome cellobiose dehydrogenase ( $\mathrm{PcCDH}$ ) anchored on a gold surface. The image frames are extracted from movies and show the time-evolution of the domain motion of PcCDH in two different concentrations of cellobiose: $0 \mu \mathrm{M}$ (left) and $150 \mu \mathrm{M}$ (right). The orange and green ovals on the right side of each set of image frames illustrate the estimated relative positions respectively of the dehydrogenase and the cytochrome domains. Reproduced with permission from reference ${ }^{[123 b]}$

\section{9- Kinetics}




\section{1- FM}

Characterizations of kinetics of single-enzyme reactions have been realized by fluorescence spectroscopy and imaging. However, in this context the enzyme was mostly confined in microdroplets, or in a gel etc. and not immobilized at a surface. We invite the interested reader to have a look at a review by Lemay and co-workers. ${ }^{[125]}$ To the best of our knowledge, very few studies have been realized in an electrochemical context. We will describe here two studies with very close configurations; however the first one is not realized under control of electrochemical potential[127] and the second is not enzymatic (only an enzymatic cofactor is considered). We believe that the two taken together could give clues about enzyme study under electrochemical potential control. The dynamics of single monomeric sarcosine oxidase (MSOX) enzymes that contain a covalently bound flavin adenine dinucleotide (FAD) cofactor which is highly fluorescent in the oxidized state and dark in the reduced state have been studied at the single-molecule level. Enzymes were confined in arrays of gold-based nanopores, and the substrate was present in solution. This configuration enabled statistical analysis of enzyme fluorescence on-off cycles over time that in turn gave access to single-enzyme rate constants. Moreover, reduction and oxidation half-reactions were shown to be independent. ${ }^{[127]}$ The second case concerns immobilized $F A D^{[128]}$ and flavin mononucleotide freely diffusing in the nanopores. ${ }^{[129]} \mathrm{A}$ similar setup allowed to study the spectroelectrochemical behavior, and especially fluorescence arising from single-electron transfer events of these two cofactors.

\section{2- SECM}

Kinetics of enzymes immobilized on an insulating surface have been more commonly resolved by SECM in enzyme-mediated feedback mode, following the pioneering work by Bard and co-workers in the 1990 's already presented in the introduction. Since then a theoretical approach based on numerical simulation has been developed to extract enzyme kinetics from SECM approach curves. ${ }^{[130]}$ We will not get into details because this goes beyond the scope of this review, although it is clear that SECM enables spatially resolved kinetics analysis. This kinetic approach can also help evaluating enzyme immobilization methods for possible applications in electrodes. For example, a GOx was entrapped in a hydrogel on $\mathrm{SiO}_{2}$ surface. The kinetics evaluation revealed a high enzyme activity, which means that the enzyme remains active and reachable by a mediator after entrapment in a polymer. The method would therefore be convenient for enzyme immobilization enabling MET on a conductive surface. ${ }^{\text {[29a] }}$ Another possibility is the mechanistic exploration of cascade enzyme-systems, with control over the distance separating the different enzymes. We describe here the study of a two-enzyme system. Acetylcholine esterase, which degrades acetylcholine into choline, was attached on a gold surface, and choline oxidase, which couples choline oxidation to $\mathrm{O}_{2}$ reduction in $\mathrm{H}_{2} \mathrm{O}_{2}$, was immobilized on the SECM 
tip. Electrochemical oxidation of $\mathrm{H}_{2} \mathrm{O}_{2}$ was then measured at the tip. Both enzymes were immobilized on SAMs by electrostatic interactions. The SECM configuration enables to control the distance between the two enzymes and thus the substrate transport. SECM experiments and numerical simulation allowed resolving the kinetics of both individual enzymes and the two-enzyme cascade. ${ }^{[131]}$

\section{Summary and outlooks}

In this review, we discussed how microscopy allows in-depth characterization of enzymatic electrodes at the local scale. We introduced different methods (SPM, fluorescence, etc...) that all come as complementary techniques to conventional electrochemistry, which on the contrary allows getting information exclusively at the scale of the entire electrode. These microscopy techniques give insight into several aspects of the enzyme behavior at the electrode surface (resp. in the electrode volume), and the obtained information has the further advantage of being spatially resolved. The number of immobilized enzymes can be estimated, as well as the dimensions of the enzyme layers or even of the single enzyme molecules. SPM gives clues about morphology, structure and conformation of the immobilized enzyme, and possibly about their motion or the evolution of the enzyme structure with time. Similarly, some mechanistic steps can be elucidated. Since local information is obtained, microscopy is ideal to realize maps of phenomena occurring at the electrode surface, either to evaluate the distribution of enzyme localization or to map enzyme activity. Finally, microscopy is the most adequate method to envision single molecule studies.

Further developments of the techniques can be envisioned since researchers not only extract various and numerous information while using standard commercial material, but also more and more tune their experimental set up to better correspond to the studied object. We quoted in this review several examples, like the case of SPM, where microelectrode tips used as UME are modified for better sensitivity or specificity. It can be a chemical modification, like Prussian blue modified SECM tip which provides a 3-order of magnitude higher selectivity for $\mathrm{H}_{2} \mathrm{O}_{2}$ reduction in the presence of oxygen compared to a platinum tip. The use of enzyme-modified tips in SECM is another strategy expected to open new insight in electrocatalysis. ${ }^{[112,131]}$ Soft probes are nowadays available: they will enable physical contact during the approach curve, without contaminating or damaging the probe or the sample, and avoid topographic artifacts. For better precision and less artifacts, different techniques are prone to be used simultaneously, thus leading to development of "hybrid techniques". To quote some examples, we described here the use of integrated SECM-AFM probes, the hybrid SICM-SECM with a nanoring electrode surrounding a nanopipette, or a technology coupling SECM to continuous nanoflow. Such multi-technique development will certainly allow in-depth understanding of complex samples. In the same idea of multiplying the amount of information, microscopy is most of the time 
not the only technique implemented for electrode characterization. It either comes in complement to other methods or has to be complemented by other methods depending on the parameters studied: ellipsometry, ATR-FTIR... Thanks to all these advances, the close future will certainly see the rise of in operando observation of enzymatic electrodes during electrochemical experiments.

\section{References}

[1] L. P. Jenner, J. N. Butt, Curr. Opin. Electrochem. 2018, 8, 81-88.

[2] I. Mazurenko, A. de Poulpiquet, E. Lojou, Curr. Opin. Electrochem. 2017, 5, 74-84.

[3] V. P. Hitaishi, R. Clement, N. Bourassin, M. Baaden, A. de Poulpiquet, S. Sacquin-Mora, A. Ciaccafava, E. Lojou, Catalysts 2018, 8.

[4] N. Mano, A. de Poulpiquet, Chem. Rev. 2018, 118, 2392-2468.

[5] C. Leger, P. Bertrand, Chem. Rev. 2008, 108, 2379-2438.

[6] I. Mazurenko, K. Monsalve, P. Infossi, M. T. Giudici-Orticoni, F. Topin, N. Mano, E. Lojou, Energy Environ. Sci. 2017, 10, 1966-1982.

[7] aX. Zhou, N. M. Andoy, G. Liu, E. Choudhary, K.-S. Han, H. Shen, P. Chen, Nat. Nanotechnol. 2012, 7, 237-241; bF. Xu, R. J. Madix, C. M. Friend, J. Am. Chem. Soc. 2018, 140, 12210-12215; cZ. Zhang, J. Kneipp, Anal. Chem. 2018, 90, 9199-9205.

[8] aT. McArdle, T. P. McNamara, F. Fei, K. Singh, C. F. Blanford, ACS Appl. Mater. Inter. 2015, 7, 25270-25280; bM. Dagys, P. Lamberg, S. Shleev, G. Niaura, I. Bachmatova, L. Marcinkeviciene, R. Meskys, J. Kulys, T. Arnebrant, T. Ruzgas, Electrochim. Acta 2014, 130, 141-147; cE. Casero, L. Vazquez, A. M. Parra-Alfambra, E. Lorenzo, Analyst 2010, 135, 1878-1903.

[9] aC. Gutierrez-Sanchez, A. Ciaccafava, P. Y. Blanchard, K. Monsalve, M. T. Giudici-Orticoni, S. Lecomte, E. Lojou, ACS Catal. 2016, 6, 5482-5492; bV. P. Hitaishi, I. Mazurenko, M. Harb, R. Clement, M. Taris, S. Castano, D. Duche, S. Lecomte, M. Ilbert, A. de Poulpiquet, E. Lojou, ACS Catal. 2018, 8, 12004-12014.

[10] K. Singh, T. McArdle, P. R. Sullivan, C. F. Blanford, Energy Environ. Sci. 2013, 6, 2460-2464.

[11] aA. Ciaccafava, P. Infossi, M. Ilbert, M. Guiral, S. Lecomte, M. T. Giudici-Orticoni, E. Lojou, Angew. Chem. Int. Ed. 2012, 51, 953-956; bM. Horch, L. Lauterbach, M. Saggu, P. Hildebrandt, F. Lendzian, R. Bittl, O. Lenz, I. Zebger, Angew. Chem. Int. Ed. 2010, 49, 8026-8029; cM. Dagys, A. Laurynenas, D. Ratautas, J. Kulys, R. Vidziunaite, M. Talaikis, G. Niaura, L. Marcinkeviciene, R. Meskys, S. Shleev, Energy Environ. Sci. 2017, 10, 498-502.

[12] G. V. Spivak, G. V. Saparin, M. V. Bykov, Soviet Physics Uspekhi-Ussr 1970, 12, 756-+.

[13] B. Liao, E. Najafi, Mater. Today Phys. 2017, 2, 46-53.

[14] G. Binnig, H. Rohrer, Ultramicroscopy 1983, 11, 157-160.

[15] D. M. Eigler, E. K. Schweizer, Nature 1990, 344, 524-526.

[16] aR. Sonnenfeld, P. K. Hansma, Science 1986, 232, 211-213; bH. Y. Liu, F. R. F. Fan, C. W. Lin, A. J. Bard, J. Am. Chem. Soc. 1986, 108, 3838-3839.

[17] N. J. Tao, C. Z. Li, H. X. He, J. Electroanal. Chem. 2000, 492, 81-93.

[18] C. J. Chen, Introduction to Scanning Tunneling Microscopy, 1993.

[19] aH. S. Park, J. H. Jang, J. Electrochem. Sci. Te. 2016, 7, 316-326; bG. Binnig, C. F. Quate, C. Gerber, Phys. Rev. Lett. 1986, 56, 930-933.

[20] A. Parra, E. Casero, L. Vazquez, J. Jin, F. Pariente, E. Lorenzo, Langmuir 2006, 22, 5443-5450.

[21] A. K. E. Ukraintsev, H. Kozak, Z. Remeš and B. Rezek, in Atomic Force Microscopy Investigations into Biology - From Cell to Protein, InTech ed., InTech, 2012, pp. 29-54.

[22] C. Wong, P. E. West, K. S. Olson, M. L. Mecartney, N. Starostina, Jom 2007, 59, 12-16.

[23] aS. Amemiya, A. J. Bard, F.-R. F. Fan, M. V. Mirkin, P. R. Unwin, in Annu. Rev. Anal. Chem., Vol. 1, 2008, pp. 95-131; bP. Sun, F. O. Laforge, M. V. Mirkin, Phys. Chem. Chem. Phys. 2007, 9, 802823. 
[24] aR. C. Engstrom, M. Weber, D. J. Wunder, R. Burgess, S. Winquist, Anal. Chem. 1986, 58, 844848; bR. C. Engstrom, T. Meaney, R. Tople, R. M. Wightman, Anal. Chem. 1987, 59, 2005-2010; CR. C. Engstrom, J. Electrochem. Soc. 1987, 134, C519-C519.

[25] aO. E. Husser, D. H. Craston, A. J. Bard, J. Electrochem. Soc. 1989, 136, 3222-3229; bD. Mandler, A. J. Bard, J. Electrochem. Soc. 1990, 137, 2468-2472; cA. J. Bard, F. R. F. Fan, D. T. Pierce, P. R. Unwin, D. O. Wipf, F. M. Zhou, Science 1991, 254, 68-74; dD. T. Pierce, P. R. Unwin, A. J. Bard, Anal. Chem. 1992, 64, 1795-1804.

[26] aL. Y. Huang, Z. Y. Li, Y. T. Lou, F. H. Cao, D. W. Zhang, X. G. Li, Materials 2018, 11; bG. Wittstock, Fresenius J. Anal. Chem. 2001, 370, 303-315.

[27] aD. Polcari, P. Dauphin-Ducharme, J. Mauzeroll, Chem. Rev. 2016, 116, 13234-13278; bC. L. Bentley, J. Edmondson, G. N. Meloni, D. Perry, V. Shkirskiy, P. R. Unwin, Anal. Chem. 2019, 91, 84-108.

[28] F. Conzuelo, A. Schulte, W. Schuhmann, P. Roy. Soc. A-Math. Phy. 2018, 474.

[29] aD. Zigah, M. Pellissier, B. Fabre, F. Barriere, P. Hapiot, J. Electroanal. Chem. 2009, 628, 144147; bM. Pellissier, D. Zigah, F. Barriere, P. Hapiot, Langmuir 2008, 24, 9089-9095.

[30] T. Wilhelm, G. Wittstock, R. Szargan, Fresenius J. Anal. Chem. 1999, 365, 163-167.

[31] aK. Eckhard, X. Chen, F. Turcu, W. Schuhmann, Phys. Chem. Chem. Phys. 2006, 8, 5359-5365; bK. Karnicka, K. Eckhard, D. A. Guschin, L. Stoica, P. J. Kulesza, W. Schuhmann, Electrochem. Commun. 2007, 9, 1998-2002.

[32] M. A. Brites Helu, H. L. Bonazza, J. L. Fernandez, J. Electroanal. Chem. 2016, 775, 64-71.

[33] G. Weber, Biochem. J. 1950, 47, 114-121.

[34] J. Eng, R. M. Lynch, R. S. Balaban, Biophys. J. 1989, 55, 621-630.

[35] aL. Bouffier, T. Doneux, Curr. Opin. Electrochem. 2017, 6, 31-37; bA. de Poulpiquet, B. Goudeau, P. Garrigue, N. Sojic, S. Arbault, T. Doneux, L. Bouffier, Chem. Sci. 2018, 9, 66226628.

[36] T. Ando, Biophys. Rev. 2018, 10, 285-292.

[37] R. A. Lazenby, R. J. White, Chemosensors 2018, 6, 28.

[38] Y. Tian, F. Yang, C. Guo, Y. Jiang, Surf. Rev. Lett. 2018, 25.

[39] J. Vangindertael, R. Camacho, W. Sempels, H. Mizuno, P. Dedecker, K. P. F. Janssen, Methods Appl. Fluoresc. 2018, 6, 54.

[40] G. Vicidomini, P. Bianchini, A. Diaspro, Nat. Methods 2018, 15, 173-182.

[41] E. Matysiak, A. M. Nowicka, B. Wagner, M. Donten, Electrochim. Acta 2016, 191, 586-593.

[42] V. Sachdeva, V. Hooda, Enzyme Microb. Tech. 2016, 89, 52-62.

[43] E. Afshari, S. Mazinani, S. O. Ranaei-Siadat, H. Ghomi, Appl. Surf. Sci. 2016, 385, 349-355.

[44] N. Maleki, S. Kashanian, E. Maleki, M. Nazari, Biochem. Eng. J. 2017, 128, 1-11.

[45] T. C. Gokoglan, S. Soylemez, M. Kesik, I. B. Dogru, O. Turel, R. Yuksel, H. E. Unalan, L. Toppare, Food Chem. 2017, 220, 299-305.

[46] Y. Yang, H. Zeng, W. S. Huo, Y. H. Zhang, J. Inorg. Organomet. P. 2017, 27, 201-214.

[47] Y. Liu, A. P. F. Turner, M. J. Zhao, W. C. Mak, Biosens. Bioelectron. 2018, 100, 374-381.

[48] S. Selvarajan, A. Suganthi, M. Rajarajan, Ultrason. Sonochem. 2018, 42, 183-192.

[49] Q. Wang, R. Xue, H. Guo, Y. L. Wei, W. Yang, J. Electroanal. Chem. 2018, 817, 184-194.

[50] C. Koch, K. Wabbel, F. J. Eber, P. Krolla-Sidenstein, C. Azucena, H. Gliemann, S. Eiben, F. Geiger, C. Wege, Front. Plant Sci. 2015, 6.

[51] A. Onoda, N. Inoue, S. Campidelli, T. Hayashi, RSC Adv. 2016, 6, 65936-65940.

[52] J. A. Guimaraes, H. C. Ferraz, T. L. M. Alves, Appl. Surf. Sci. 2014, 298, 68-74.

[53] M. David, M. M. Barsan, C. M. A. Brett, M. Florescu, Sensor Actuat. B-Chem. 2018, 255, 32273234.

[54] M. B. Fritzen-Garcia, V. C. Zoldan, I. Oliveira, V. Soldi, A. A. Pasa, T. B. Creczynski-Pasa, Biotechnol. Bioeng. 2013, 110, 374-382.

[55] T. Mohan, R. Rathner, D. Reishofer, M. Koller, T. Elschner, S. Spirk, T. Heinze, K. StanaKleinschek, R. Kargl, Biomacromolecules 2015, 16, 2403-2411. 
[56] H. Akbulut, G. Bozokalfa, D. N. Asker, B. Demir, E. Guler, D. O. Demirkol, S. Tirnur, Y. Yagci, ACS Appl. Mater. Inter. 2015, 7, 20612-20622.

[57] Y. R. C. Urena, P. N. Lisboa, M. Szardenings, L. Gatjen, P. L. M. Noeske, K. Rischka, Appl. Surf. Sci. 2016, 385, 216-224.

[58] B. Wongkaew, P. Wongkaew, P. Thanutong, ChitsanuphongThanutong, Int. J. Geomate 2018, 14, 132-139.

[59] C. Vaz-Dominguez, M. Pita, A. L. de Lacey, S. Shleev, A. Cuesta, J. Phys. Chem. C 2012, 116, 16532-16540.

[60] E. M. Oliveira, S. Beyer, J. Heinze, Bioelectrochemistry 2007, 71, 186-191.

[61] Y. Inoue, M. Saito, H. Yoshikawa, E. Tamiya, Electrochim. Acta 2017, 240, 447-455.

[62] R. Cai, S. Abdellaoui, J. P. Kitt, C. Irvine, J. M. Harris, S. D. Minteer, C. Korzeniewski, Anal. Chem. 2017, 89, 13290-13298.

[63] W. W. Chen, W. S. Yang, Y. L. Lu, W. J. Zhu, X. Chen, Anal. Methods 2017, 9, 3213-3220.

[64] A. Kuchler, M. Yoshimoto, S. Luginbuhl, F. Mavelli, P. Walde, Nat. Nanotechnol. 2016, 11, 409420.

[65] K. A. Vincent, A. Parkin, F. A. Armstrong, Chem. Rev. 2007, 107, 4366-4413.

[66] N. Patel, M. C. Davies, R. J. Heaton, C. J. Roberts, S. J. B. Tendler, P. M. Williams, Appl. Phys. AMater. 1998, 66, S569-S574.

[67] D. Pankratov, J. Sotres, A. Barrantes, T. Arnebrant, S. Shleev, Langmuir 2014, 30, 2943-2951.

[68] N. Heidary, T. Utesch, M. Zerball, M. Horch, D. Millo, J. Fritsch, O. Lenz, R. von Klitzing, P. Hildebrandt, A. Fischer, M. A. Mroginski, I. Zebger, Plos One 2015, 10.

[69] E. Casero, M. D. Petit-Dominguez, L. Vazquez, I. Ramirez-Asperilla, A. M. Parra-Alfambra, F. Pariente, E. Lorenzo, Talanta 2013, 115, 401-408.

[70] B. Gunasekera, C. Abou Diwan, G. Altawallbeh, H. Kalil, S. Maher, S. Xu, M. Bayachou, ACS Appl. Mater. Inter. 2018, 10, 7745-7755.

[71] A. N. Patel, A. Anne, A. Chovin, C. Demaille, E. Grelet, T. Michon, C. Taofifenua, Small 2017, 13.

[72] Q. J. Chi, J. D. Zhang, P. S. Jensen, H. E. M. Christensen, J. Ulstrup, Faraday Discuss. 2006, 131, 181-195.

[73] J. D. Gwyer, J. Zhang, J. N. Butt, J. Ulstrupy, Biophys. J. 2006, 91, 3897-3906.

[74] V. Climent, Y. Fu, S. Chumillas, B. Maestro, J.-F. Li, A. Kuzume, S. Keller, T. Wandlowski, J. Phys. Chem. C 2014, 118, 15754-15765.

[75] A. Anne, A. Chovin, C. Demaille, M. Lafouresse, Anal. Chem. 2011, 83, 7924-7932.

[76] A. Blout, F. Billon, C. Calers, C. Methivier, A. Pailleret, H. Perrot, C. Jolivalt, Electrochim. Acta 2018, 277, 255-267.

[77] M. L. Cortez, W. Marmisolle, D. Pallarola, L. I. Pietrasanta, D. H. Murgida, M. Ceolin, O. Azzaroni, F. Battaglini, Chem.-Eur. J. 2014, 20, 13366-13374.

[78] Y. Kikkawa, K. Yamashita, T. Hiraishi, M. Kanesato, Y. Doi, Biomacromolecules 2005, 6, 20842090.

[79] aA. Konash, M. J. Cooney, B. Y. Liaw, D. M. Jameson, J. Mater. Chem. 2006, 16, 4107-4109; bG. L. Martin, S. D. Minteer, M. J. Cooney, ACS Appl. Mater. Inter. 2009, 1, 367-372.

[80] C. Mateo-Mateo, A. S. Michardiere, S. Gounel, I. Ly, J. Rouhana, P. Poulin, N. Mano, Chemelectrochem 2015, 2, 1908-1912.

[81] F. Wu, S. D. Minteer, Biomacromolecules 2013, 14, 2739-2749.

[82] G. L. Martin, S. D. Minteer, M. Cooney, Chem. Commun. 2011, 47, 2083-2085.

[83] D. M. Chudakov, M. V. Matz, S. Lukyanov, K. A. Lukyanov, Physiol. Rev. 2010, 90, 1103-1163.

[84] C. Leger, A. K. Jones, S. P. J. Albracht, F. A. Armstrong, J. Phys. Chem. B 2002, 106, 13058-13063.

[85] C. C. Page, C. C. Moser, X. X. Chen, P. L. Dutton, Nature 1999, 402, 47-52.

[86] S. V. Hexter, T. F. Esterle, F. A. Armstrong, Phys. Chem. Chem. Phys. 2014, 16, 11822-11833.

[87] P. Olejnik, A. Pawlowska, B. Palys, Electrochim. Acta 2013, 110, 105-111.

[88] M. Bergkvist, J. Carlsson, S. Oscarsson, J. Phys. Chem. B 2001, 105, 2062-2069.

[89] H. Muguruma, Y. Kase, N. Murata, K. Matsumura, J. Phys. Chem. B 2006, 110, 26033-26039. 
[90] J. M. Abad, M. Velez, C. Santamaria, J. M. Guisan, P. R. Matheus, L. Vazquez, I. Gazaryan, L. Gorton, T. Gibson, V. M. Fernandez, J. Am. Chem. Soc. 2002, 124, 12845-12853.

[91] E. Casero, A. M. G. de Quesada, J. Jin, M. C. Quintana, F. Pariente, H. D. Abruna, L. Vazquez, E. Lorenzo, Anal. Chem. 2006, 78, 530-537.

[92] A. V. Kartashov, G. Serafini, M. Dong, S. Shipovskov, I. Gazaryan, F. Besenbacher, E. E. Ferapontova, Phys. Chem. Chem. Phys. 2010, 12, 10098-10107.

[93] C. Gutierrez-Sanchez, D. Olea, M. Marques, V. M. Fernandez, I. A. C. Pereira, M. Velez, A. L. De Lacey, Langmuir 2011, 27, 6449-6457.

[94] O. Gutierrez-Sanz, P. Natale, I. Marquez, M. C. Marques, S. Zacarias, M. Pita, I. A. C. Pereira, I. Lopez-Montero, A. L. De Lacey, M. Velez, Angew. Chem. Int. Ed. 2016, 55, 6216-6220.

[95] O. Gutierrez-Sanz, D. Olea, M. Pita, A. P. Batista, A. Alonso, M. M. Pereira, M. Velez, A. L. De Lacey, Langmuir 2014, 30, 9007-9015.

[96] aE. Matysiak, A. J. R. Botz, J. Clausmeyer, B. Wagner, W. Schuhmann, Z. Stojek, A. M. Nowicka, Langmuir 2015, 31, 8176-8183; bC. A. Gunawan, E. V. Nam, C. P. Marquis, J. J. Gooding, P. Thordarson, C. Zhao, Chemelectrochem 2016, 3, 1150-1156.

[97] L. Alfonta, B. Meckes, L. Amir, O. Schlesinger, S. Ramachandran, R. Lal, Anal. Chem. 2014, 86, 7674-7680.

[98] aX. Zhang, F. Sun, X. Peng, W. Jin, Anal. Chem. 2007, 79, 1256-1261; bN. Gao, X. Wang, L. Li, X. Zhang, W. Jin, Analyst 2007, 132, 1139-1146.

[99] J. F. Zhou, C. Campbell, A. Heller, A. J. Bard, Anal. Chem. 2002, 74, 4007-4010.

[100] C. A. Zhao, G. Wittstock, Anal. Chem. 2004, 76, 3145-3154.

[101] W. Nogala, K. Szot, M. Burchardt, F. Roelfs, J. Rogalski, M. Opallo, G. Wittstock, Analyst 2010, 135, 2051-2058.

[102] Y. Takahashi, A. I. Shevchuk, P. Novak, Y. Murakami, H. Shiku, Y. E. Korchev, T. Matsue, J. Am. Chem. Soc. 2010, 132, 10118-10126.

[103] P. Wilde, T. Quast, H. B. Aiyappa, A. Botz, T. Tarnev, M. Marquitan, S. Feldhege, A. Lindner, C. Andronescu, W. Schuhmann, Chemelectrochem 2018, 5, 3083-3088.

[104] J. M. Abad, A. Y. Tesio, E. Martinez-Perinan, F. Pariente, E. Lorenzo, Nano Research 2018, 11, 4232-4244.

[105] G. Wittstock, W. Schuhmann, Anal. Chem. 1997, 69, 5059-5066.

[106] T. Wilhelm, G. Wittstock, Langmuir 2002, 18, 9485-9493.

[107] J. L. Fernandez, N. Mano, A. Heller, A. J. Bard, Angew. Chem. Int. Ed. 2004, 43, 6355-6357.

[108] M. Ciobanu, H. A. Kincaid, G. K. Jennings, D. E. Cliffel, Langmuir 2005, 21, 692-698.

[109] P. Vatsyayan, C. Iffelsberger, C. C. Mayorga-Martinez, F.-M. Matysik, Anal. Methods 2016, 8, 6847-6855.

[110] J. Li, J. Yu, Bioelectrochemistry 2008, 72, 102-106.

[111] M. M. Pribil, F. Cortes-Salazar, E. A. Andreyev, A. Lesch, E. E. Karyakina, O. G. Voronin, H. H. Girault, A. A. Karyakin, J. Electroanal. Chem. 2014, 731, 112-118.

[112] F. Zhao, F. Conzuelo, V. Hartmann, H. Li, S. Stapf, M. M. Nowaczyk, M. Roegner, N. Plumere, W. Lubitz, W. Schuhmann, Biosens. Bioelectron. 2017, 94, 433-437.

[113] I. Morkvenaite-Vilkonciene, A. Ramanaviciene, A. Ramanavicius, RSC Adv. 2014, 4, 5006450069.

[114] C. Kranz, G. Friedbacher, B. Mizaikoff, Anal. Chem. 2001, 73, 2491-+.

[115] C. Kranz, A. Kueng, A. Lugstein, E. Bertagnolli, B. Mizaikoff, Ultramicroscopy 2004, 100, 127134.

[116] D. A. Walsh, J. L. Fernandez, J. Mauzeroll, A. J. Bard, Anal. Chem. 2005, 77, 5182-5188.

[117] T. Kai, S. Chen, E. Monterroso, F. Zhou, Anal. Chem. 2015, 87, 4523-4529.

[118] J. C. O'Brien, J. Shumaker-Parry, R. C. Engstrom, Anal. Chem. 1998, 70, 1307-1311.

[119] R. Lei, L. Stratmann, D. Schaefer, T. Erichsen, S. Neugebauer, N. Li, W. Schuhmann, Anal. Chem. 2009, 81, 5070-5074.

[120] K. Szot, W. Nogala, J. Niedziolka-Joensson, M. Joensson-Niedziolka, F. Marken, J. Rogalski, C. N. Kirchner, G. Wittstock, M. Opallo, Electrochim. Acta 2009, 54, 4620-4625. 
[121] W. Nogala, M. Burchardt, M. Opallo, J. Rogalski, G. Wittstock, Bioelectrochemistry 2008, 72, 174-182.

[122] V. Climent, J. Zhang, E. P. Friis, L. H. Ostergaard, J. Ulstrup, J. Phys. Chem. C 2012, 116, 12321243.

[123] aX. Hao, J. Zhang, H. E. M. Christensen, H. Wang, J. Ulstrup, Chemphyschem 2012, 13, 29192924; bH. Harada, A. Onoda, T. Uchihashi, H. Watanabe, N. Sunagawa, M. Samejima, K. Igarashi, T. Hayashi, Chem. Sci. 2017, 8, 6561-6565.

[124] J. Zhang, Q. Chi, A. G. Hansen, P. S. Jensen, P. Salvatore, J. Ulstrup, Febs Letters 2012, 586, 526535.

[125] K. Mathwig, Q. Chi, S. G. Lemay, L. Rassaei, Chemphyschem 2016, 17, 452-457.

[126] J. D. Zhang, A. C. Welinder, A. G. Hansen, H. E. M. Christensen, J. Ulstrup, J. Phys. Chem. B 2003, 107, 12480-12484.

[127] J. Zhao, S. P. Branagan, P. W. Bohn, Appl. Spectrosc. 2012, 66, 163-169.

[128] J. Zhao, L. P. Zaino, P. W. Bohn, Faraday Discuss. 2013, 164, 57-69.

[129] L. P. Zaino, D. A. Grismer, D. Han, G. M. Crouch, P. W. Bohn, Faraday Discuss. 2015, 184, 101115.

[130] R. Cornut, P. Hapiot, C. Lefrou, J. Electroanal. Chem. 2009, 633, 221-227.

[131] Z.-Q. Wu, W.-Z. Jia, K. Wang, J.-J. Xu, H.-Y. Chen, X.-H. Xia, Anal. Chem. 2012, 84, 10586-10592.

\section{Keywords :}

Enzyme, electrode, electrochemistry, microscopy, scanning probe microscopy, AFM, SECM, STM, fluorescence

\section{Table of content :}

Local characterization: In bioelectrochemical applications such as biosensors and biofuel cells, redox enzymes are immobilized at electrodes which collect or provide the required electrons. Various electrochemical techniques allow understanding the behaviour of the enzymatic electrodes at the global scale. This review describes how scanning probe microscopy (AFM, STM, SECM) and fluorescence microscopy are used to obtain complementary information at the local scale.

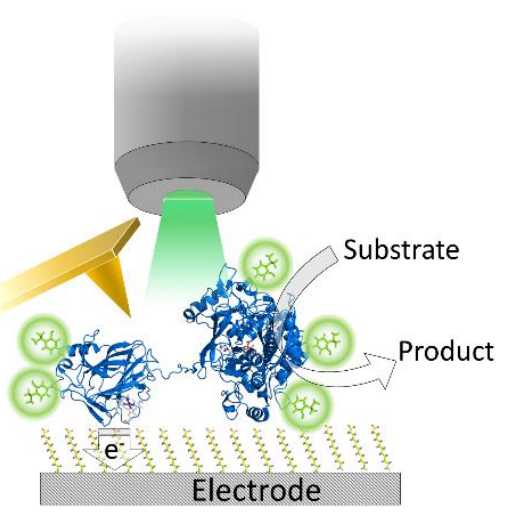

